\title{
A Multiple-Doppler Synthesis and Continuity Adjustment Technique (MUSCAT) to Recover Wind Components from Doppler Radar Measurements
}

\author{
Olivier Bousquet AND Michel ChONG \\ Centre National de Recherches Météorologiques, Météo-France and CNRS, Toulouse, France
}

(Manuscript received 20 January 1997, in final form 23 May 1997)

ABSTRACT

\begin{abstract}
A new approach is presented to account for a simultaneous solution of the three wind components from at least a pair of Doppler radar observations, which could remove potential drawbacks of an iterative (nonsimultaneous) solution of Cartesian dual-Doppler analysis techniques. The multiple-Doppler synthesis and continuity adjustment technique (MUSCAT) is derived from the extended overdetermined dual-Doppler (EODD) variational formalism that contains the basis for a simultaneous (noniterative) solution of a dual- or multiple-equation system and a mass continuity equation. Necessary accommodations are discussed, including the solutions for a planeto-plane synthesis (as in EODD) instead of a fully three-dimensional and computationally intensive analysis, owing to the three-dimensional character of the continuity equation. The evaluation of MUSCAT is carried out by first considering real data and then performing numerical tests based on simulated radar observations. The comparative study with EODD applications shows that MUSCAT provides a more regular description of the airflow and that EODD may still contain residual errors that make the retrieved wind components inconsistent. Results from the numerical tests definitely reveal the real improvements of MUSCAT in synthesizing Doppler radar data.
\end{abstract}

\section{Introduction}

Recently, Chong and Testud (1996) and Chong and Campos (1996) investigated the potential of, respectively, coplane and Cartesian dual-Doppler analyses in deducing the wind components from airborne Doppler radars that are equipped to make fore-aft observations and thus to collect dual-Doppler data from a single straight flight path, as proposed by Frush et al. (1986). Both approaches combine the dual-Doppler observations with the anelastic mass continuity equation and consider empirical relationships between radar reflectivity and terminal fallspeed of precipitating particles to correct for their contribution to the Doppler measurements.

The coplane analysis proceeds in a well-posed solution in the mathematical sense, since it uses the natural cylindrical frame attached to the radar scanning that yields an unambiguous determination of two orthogonal cylindrical components (say, the coplanar components) from observations, which are subsequently utilized in the continuity equation to deduce the third component. This is a major advantage over the conventional Cartesian dual-Doppler analysis in which the dual-equation system is dependent on the three wind components. Un-

Corresponding author address: Dr. Michel Chong, CNRM, 42 Avenue Coriolis, 31057 Toulouse, Cedex, France.

E-mail: chong@meteo.fr less simultaneously solving this system with the continuity equation, an iterative procedure is required to determine the horizontal wind components, on the one hand, and the vertical component, on the other hand. Usually, coplane and Cartesian dual-Doppler techniques are limited to low elevation angles $\left(\leq 45^{\circ}\right)$ on either side of the aircraft in order to mitigate the contribution of the vertical particle motions to the observed Doppler velocities. Moreover, at higher elevation angles, the Cartesian wind synthesis tends to be ill conditioned, as shown by Chong and Campos (1996). These limitations prevent wind synthesis in a cone above and below the aircraft and also prevent taking advantage of the full radar coverage when the aircraft is penetrating into the cloud system.

The extended overdetermined dual-Doppler (EODD) technique, performed in a Cartesian frame and proposed by Chong and Campos (1996), is an alternative to overcome the limitation problems. The proposed variational formalism applies to dual- and multiple-Doppler datasets, such as quad-Doppler observations (Jorgensen et al. 1996) that could be collected from coordinated and parallel flight tracks in a two-plane mission. Its application to datasets obtained during the Tropical Ocean Global Atmosphere Coupled Ocean-Atmosphere Response Experiment (TOGA COARE) (Webster and Lukas 1992) field project in the western Pacific warm pool has shown that a highly reliable flow structure could be obtained in regions where the traditional approach pro- 
duced unrealistic components. However, due to a nonsimultaneous solution of the wind components (as in the conventional analysis, an iterative process is needed), we suspect that residual errors may remain.

This paper proposes a new approach to account for a simultaneous solution, based upon the EODD variational formalism and valid for at least dual-Doppler observations. An overview of EODD and its potential limits is given in section 2. Section 3 presents the proposed multiple-Doppler synthesis and continuity adjustment technique (MUSCAT), including an analysis of its numerical aspects that are detailed in appendixes A-D. The evaluation of MUSCAT is carried out in section 4, based on a comparative study with respect to EODD. Data from TOGA COARE, previously used to test EODD, and simulated radar observations are employed to investigate the performances of MUSCAT.

\section{Overview of EODD analysis}

\section{a. Formalism}

The aim of this section is not to recall the reasons that have motivated the development of EODD analysis, as can be found in Chong and Campos (1996), but to remind the reader of its main characteristics. EODD is a variational adjustment of the horizontal Cartesian wind components $u$ and $v$ (considering a specified vertical velocity $w^{0}$ ) to observed Doppler velocities in individual horizontal planes, which minimizes the functional

$$
\begin{gathered}
F(u, v)=\int_{S} \underbrace{\mathrm{A}}_{\sum_{i}\left[\alpha_{i} u+\beta_{i} v+\gamma_{i}\left(w^{0}+v_{T}\right)-V_{i}\right]^{2}} \\
+\underbrace{\mu_{1}\left[\frac{\partial u}{\partial x}+\frac{\partial v}{\partial y}+\frac{\partial w^{0}}{\partial z}-\kappa w^{0}\right]^{2}}_{\mathrm{C}} \\
+\underbrace{\mathrm{B}}_{\left.\mu_{2}\left[J_{2}(u)+J_{2}(v)\right]\right\}}\} d x d y
\end{gathered}
$$

according to

$$
\frac{\partial F}{\partial u}=0 \quad \text { and } \quad \frac{\partial F}{\partial v}=0
$$

and includes the airmass continuity equation to derive the vertical velocity $w$,

$$
\partial u / \partial x+\partial v / \partial y+\partial w / \partial z-\kappa w=0
$$

Subscript $i$ defines the radar (or beam for dual-beam airborne radars) number that ranges from 1 to at least 2. Here, $v_{T}$ is the terminal fallspeed of precipitation particles that can be estimated from an empirical rela- tionship with the observed radar reflectivity; $\alpha_{i}, \beta_{i}, \gamma_{i}$ are the direction cosines, in the $x, y$, and $z$ directions, respectively, of the beam-pointing angles; and $V_{i}$ is the radial velocity from radar $i$. Here, $J_{2} \equiv\left(\partial^{2} / \partial x^{2}\right)^{2}+2\left(\partial^{2} /\right.$ $\partial x \partial y)^{2}+\left(\partial^{2} / \partial y^{2}\right)^{2}$ is a differential operator, $\kappa=$ $-\partial \ln \rho / \partial z$ accounts for the air density $(\rho)$ decrease with height, $\mu_{1}$ and $\mu_{2}$ are normalized weighting parameters that control the relative importance of terms $\mathrm{B}$ and $\mathrm{C}$ with respect to A. As in the conventional dual-Doppler analysis, solution of (2) is obtained through an iterative process with (3), allowing a step-by-step correction of the vertical air velocity contribution to the measured radial velocity. [At the initial step, $w^{0}=0$ in (1); then it is the solution of (3).]

In (1), A is the data adjustment term that links the three wind components to the measured radial velocities. This is the classic least squares minimization formalism of the overdetermined dual-Doppler (ODD) technique (Ray and Sangren 1983), which leads to an equivalent form of the conventional dual-equation system. Term B is the least squares expression of the mass continuity equation, which limits the extent of horizontal wind variations through the divergence term during the iterations. In essence, it allows us to moderate the contribution of errors in determining $w$ from (3) and thus to improve the convergence of the iterative process. Term $\mathrm{C}$ is a constraint on second derivatives of $u$ and $v$ and helps to provide regular horizontal wind field by filtering out small-scale variations. A main advantage of this term is to give an objective solution of the illconditioned dual-Doppler analysis that can be caused by the geometrical configuration of the Doppler measurements in both the cone above and below the aircraft that involves high elevation angles. Note that such illconditioned analysis also occurs in regions close to the radar baseline when two ground-based radars are involved. In this paper, discussion concerning airborne radar analysis can be readily extended to the case of ground-based radars.

\section{b. Limitations of EODD}

Although the variational EODD technique was shown to provide highly reliable flow structure in regions where the traditional approach failed, there remain some limitations that should be clarified in this paper and that justify additional improvements. The first limitation concerns the interpolation of the polar coordinate radar data onto a Cartesian grid, prior to the application of EODD, in order to obtain, at each grid point, one Doppler-radial vector for each radar (or beam). Interpolated grid values are defined as distance-weighted averages of all data falling inside an ellipsoid with an influence radius in the horizontal twice as large as in the vertical. A fixed interpolation function applies at short and long ranges differently. At short range this function forces averaging vectors pointing in quite different directions and creates a sampling problem, as these data are dif- 
ferent due to the changes in elevation and/or azimuth angles and, hence, should not be averaged. In the context of airborne radar sampling, which consists of helical scans about the aircraft axis, the averaging process involves such a variety of viewing angles for grid points close to this axis, more specifically above and below it, because of the larger horizontal influence radius. As a result, the interpolated radial velocities and the associated beam-pointing angles are less accurate in these regions than elsewhere, exacerbating the errors in estimating the horizontal components. A solution would consist of incorporating the interpolation step into the least squares data adjustment procedure, allowing the interpolation to take account of a number of measurements (i.e., all the available observed velocity vectors and their actual orientation) instead of a unique mean observation from each radar or beam.

The second limitation results from the actual limits of the proposed approach. Let us examine the functional $F$ [Eq. (1)] to be minimized. Constraint $\mathrm{C}$ on the secondorder derivatives of the wind field, which acts as a lowpass filter, basically applies to the results of the least squares data adjustment given by term $\mathrm{A}$ in order to correct or reduce unreasonable horizontal winds that can be obtained near the flight track (see Fig. 7 in Chong and Campos 1996). However, in some circumstances, this correction may be incomplete. Consider that unstable solutions occur within an interval of $4 \Delta x$ in the cross-track direction, where $\Delta x$ is the grid spacing. If their differences with solutions from well-defined regions are maximized at the center of the unstable zone, then they may be interpreted as varying with an equivalent wavelength greater than or equal to $4 \Delta x$. Because the cutoff wavelength of the low-pass filter (defined as the wavelength for which the filter response is 0.5 since the response function is not a pure step function) is usually $4 \Delta x$, the filtered winds may contain residuals that can reach $50 \%$ of the amplitude of the wind bias. As a consequence, a localized wind extremum is obtained and causes an artificial convergence/divergence doublet that is penalizing for the determination of $w$ through (3).

The above limitation concurrently combines with the problem arising from a separate estimation of the horizontal and vertical wind components. Indeed, the iterative process forms another important limitation of EODD in this region of unstable solution near the flight track, where vertical motion accounts contribute more than $70 \%$ of the measured radial velocity at elevation angles greater than $45^{\circ}$. In some conditions, prescribing $w^{0}=0$ in the initial step can be excessive, and biased $u$ and $v$ estimates can be obtained due to associating nonconsistent vertical and horizontal velocities during the iterative process.

In fact, the dual-Doppler equation system and the continuity equation define a mathematically complete system to retrieve the three wind components, and illconditioned problems could disappear if they are solved simultaneously (noniterative process). Such a solution has been already investigated by Scialom and Lemaître (1990) in their adjustment technique of observations with an analytical wind field and more recently by Gamache (1995), who found, however, that the technique was computationally intensive and required high memory storage in a three-dimensional context. An alternative approach consists of resolving the above-mentioned equations in individual planes. The EODD approach offers such an opportunity by simply extending the minimization problem to the variable $w$. Improvements are, however, necessary to preserve the plane-toplane wind synthesis. These will be described in the next section, which presents the basis for MUSCAT, a method that can be applied to airborne or ground-based radar data.

\section{MUSCAT formulation}

The variational expression (1) for individual planes can be rewritten in a generalized form including the vertical air velocity $w$ as

$$
\begin{gathered}
F^{\prime}(u, v, w)=\int_{S}\left[\mathrm{~A}^{\prime}(u, v, w)+\mathrm{B}^{\prime}(u, v, w)\right. \\
\left.+\mathrm{C}^{\prime}(u, v, w)\right] d x d y
\end{gathered}
$$

where $\mathrm{A}^{\prime}, \mathrm{B}^{\prime}$, and $\mathrm{C}^{\prime}$ are expressions having equivalent meanings as $\mathrm{A}, \mathrm{B}$, and $\mathrm{C}$ in (1). These expressions, hereafter described along with their numerical counterpart, are the MUSCAT formulation of data fit, continuity equation adjustment, and filtering process, respectively. Solution for $u, v$, and $w$ is derived according to a simultaneous resolution of

$$
\frac{\partial F^{\prime}}{\partial u}=0, \quad \frac{\partial F^{\prime}}{\partial v}=0, \quad \text { and } \quad \frac{\partial F^{\prime}}{\partial w}=0
$$

\section{a. Data fit}

As previously explained, the use of grid values, obtained with a fixed interpolation function, in the data fit expression A of (1) may cause problems in regions above and below the aircraft. Because data distribution is not coincident with the grid mesh used to recover the three-dimensional wind field, the interpolation process must be maintained. The alternative proposed in the previous section, consisting of inclusion of this process in the data fit, may be expressed for a grid point $(i, j)$ of a horizontal plane as

$$
\begin{aligned}
& \mathrm{A}_{i j}^{\prime}(u, v, w) \\
& \quad=\frac{1}{N} \sum_{p=1}^{n_{p}} \sum_{q=1}^{n_{q}(p)} \omega_{q}\left[\alpha_{q} u+\beta_{q} v+\gamma_{q}\left(w+v_{T}\right)-V_{q}\right]^{2},
\end{aligned}
$$

where $u, v, w$, and $v_{T}$ are defined for the considered 
grid point; subscript $q$ defines the $q$ th measurement of a total number $n_{q}$ that can be observed from the $p$ th radar and that falls inside an ellipsoid of influence centered on the grid point; $N$ is the cumulated $n_{q}$ 's over the considered domain; $\omega_{q}$ is the Cressman weighting function depending on the distance between measurement $q$ within the ellipsoid and grid point $\left(\omega_{q}=1\right.$ at the center of the ellipsoid and $\omega_{q}=0$ on its surface); and $n_{p}$ is the total number of radars that must be equal to at least 2. Weighted data fit was also used by Roux and Sun (1990) to evaluate wind components from a series of conical single-Doppler scans of ground-based radar.

In (6), the terminal particle fallspeed $v_{T}$ is evaluated from preinterpolated radar reflectivity $Z$, instead of individual reflectivity observations, because the radar signal may undergo strong attenuation in regions of heavy precipitations and, to further mitigate this effect, the maximum gridpoint value from more than two radars can be considered. Another possibility may be provided by the stereoradar analysis of dual reflectivity observations proposed to estimate corrected reflectivity fields (Testud and Amayenc 1989; Kabèche and Testud 1995).

Minimizing (6) with respect to $u, v$, and $w$ requires, for each radar dataset, the estimation of nine terms for each individual grid point. They are defined as follows:

$$
\begin{aligned}
M_{u u} & =\frac{1}{N} \sum_{q} \omega_{q} \alpha_{q}^{2}, \\
M_{v v} & =\frac{1}{N} \sum_{q} \omega_{q} \beta_{q}^{2}, \\
M_{w w} & =\frac{1}{N} \sum_{q} \omega_{q} \gamma_{q}^{2}, \\
M_{u v} & =\frac{1}{N} \sum_{q} \omega_{q} \alpha_{q} \beta_{q}, \\
M_{u w} & =\frac{1}{N} \sum_{q} \omega_{q} \alpha_{q} \gamma_{q}, \\
M_{v w} & =\frac{1}{N} \sum_{q} \omega_{q} \beta_{q} \gamma_{q}, \\
P_{u} & =\frac{1}{N} \sum_{q} \omega_{q} \alpha_{q} V_{q}, \\
P_{v} & =\frac{1}{N} \sum_{q} \omega_{q} \beta_{q} V_{q},
\end{aligned}
$$

and

$$
P_{w}=\frac{1}{N} \sum_{q} \omega_{q}\left(\gamma_{q} V_{q}-\gamma_{q}^{2} v_{T}\right),
$$

such that

$$
\left[\begin{array}{ccc}
\sum M_{u u} & \sum M_{u v} & \sum M_{u w} \\
\sum M_{u v} & \sum M_{v v} & \sum M_{v w} \\
\sum M_{u w} & \sum M_{v w} & \sum M_{w w}
\end{array}\right]\left[\begin{array}{l}
u \\
v \\
w
\end{array}\right]=\left[\begin{array}{c}
\sum P_{u} \\
\sum P_{v} \\
\sum P_{w}
\end{array}\right] .
$$

Summations in the matrix arrays are performed over the number $n_{p}(\geq 2)$ of available radars that contribute to the observations at the considered grid point. Because $v_{T}$ is derived from an independent processing and is a constant during the summation, term $P_{w}$ can be split into two terms:

$$
P_{w}^{\prime}=\frac{1}{N} \sum \omega_{q} \gamma_{q} V_{q}
$$

and

$$
P_{w}^{\prime \prime}=\frac{1}{N} \sum \omega_{q} \gamma_{q}^{2},
$$

where $P_{\omega}=P_{\omega}^{\prime}-P_{\omega}^{\prime \prime} v_{T}$. It should be noted that if the $v_{T}-Z$ relationship is changed, the above terms need not be reevaluated, as long as these are saved. This is what is done for MUSCAT software.

\section{b. Continuity equation adjustment}

The adjustment term $\mathrm{B}^{\prime}$ for the continuity equation is exactly the same as in the EODD approach and is rewritten as

$$
\mathrm{B}^{\prime}(u, v, w)=\mu_{1}\left(\frac{\partial u}{\partial x}+\frac{\partial v}{\partial y}+\frac{1}{\rho} \frac{\partial \rho w}{\partial z}\right)^{2} .
$$

In essence, $\mathrm{B}^{\prime}$ must be discretized by considering grid point values of $u, v$, and $w$. Due to the vertical derivative term, this necessarily involves two successive planes. The discrete form of (9) allowing a plane-to-plane formulation is described in appendix A. An off-centered finite-difference scheme is used to express $\mathrm{B}^{\prime}$ at the center of a grid box in terms of net mass flux through the box sides. This differentiating scheme accounts for the boundary conditions at the surface when starting the wind synthesis from the lowest level, or at the top in a downward plane-to-plane analysis, so that the integration of the continuity equation is implicitly performed.

\section{c. Filtering process}

As in EODD, the role of term $C^{\prime}$ in (4) is to smooth out small-scale variations of $u, v$, and also $w$ in the horizontal. Considering $\mathrm{B}^{\prime}$ as a weak constraint, it can be shown (appendix B) that minimizing second derivatives of wind components through $J_{2}(u), J_{2}(v)$, and $J_{2}(w)$ with a weight $\mu_{2}$ as in (1) is equivalent to a lowpass filter defined by a simple transfer function,

$$
\mathrm{TF}=\frac{1}{1+\mu_{2}|\mathbf{k}|^{4}},
$$

where $\mathbf{k}=\left(k_{x}, k_{y}\right)$ is the wave vector in the $x-y$ plane. Such a transfer function is isotropic and has a $3-\mathrm{dB}$ cutoff wavenumber $k_{c}$ controlled by $\mu_{2}$ according to $k_{c}$ $=\mu_{2}^{-1 / 4}$, that is, a cutoff wavelength $\lambda_{c}=2 \pi \mu_{2}^{1 / 4}$.

In fact, the adjustment for the continuity equation cannot be considered as a simple constraint since it is 
an integral part of MUSCAT (in contrast to EODD). Therefore, the transfer function is no longer isotropic, as shown in appendix B. Additional terms to $J_{2}(u)$, $J_{2}(v)$, and $J_{2}(w)$ are then required to obtain an isotropic response of the filtering process, which are

$$
\begin{aligned}
& J_{2}^{\prime}(u)=\mu_{1}\left[\left(\partial^{3} u / \partial x^{3}\right)^{2}+2\left(\partial^{3} u / \partial x^{2} \partial y\right)^{2}+\left(\partial^{3} u / \partial x \partial y^{2}\right)^{2}\right], \\
& J_{2}^{\prime}(v)=\mu_{1}\left[\left(\partial^{3} v / \partial y^{3}\right)^{2}+2\left(\partial^{3} v / \partial x \partial y^{2}\right)^{2}+\left(\partial^{3} v / \partial x^{2} \partial y\right)^{2}\right],
\end{aligned}
$$

and

$$
\begin{gathered}
J_{2}^{\prime}(w)=\mu_{1}\left[\left(\partial^{3} w / \partial x^{2} \partial z\right)^{2}+\left(\partial^{3} w / \partial y^{2} \partial z\right)^{2}\right. \\
\left.+2\left(\partial^{3} w / \partial x \partial y \partial z\right)^{2}\right] .
\end{gathered}
$$

Finally, term $\mathrm{C}^{\prime}$ is given by

$$
\begin{gathered}
\mathrm{C}^{\prime}(u, v, w)=\mu_{2}\left[J_{2}(u)+J_{2}^{\prime}(u)+J_{2}(v)+J_{2}^{\prime}(v)\right. \\
\left.+J_{2}(w)+J_{2}^{\prime}(w)\right] .
\end{gathered}
$$

The discrete forms of the various derivatives involved in the expression of $J_{2}$ and $J_{2}^{\prime}$ are developed in appendix C.

\section{d. Resolution procedure}

Appendix D details the matrix equation that derives from (5), taking account of the explicit expressions and discretisized forms of $\mathrm{A}^{\prime}, \mathrm{B}^{\prime}$, and $\mathrm{C}^{\prime}$. Considering an $m \times n$ Cartesian grid on the horizontal, the final form of the variational adjustment of $u, v$, and $w$ is given by the matrix equation

$$
\mathbf{M U}=\mathbf{P},
$$

where $\mathbf{M}$ is a $(3 m n)^{2}$ band-diagonal, positive definite symmetric matrix, composed of 61 diagonals into 19 groups; $\mathbf{U}$ is a $(3 m n)$ unique vector composed of three successive vectors containing the $u, v$, and $w$ grid elements; and $\mathbf{P}$ is a ( $3 m n)$ vector depending on known parameters (observations, boundary conditions). Matrix $\mathbf{M}$ and vector $\mathbf{P}$ are first filled with observational terms defined in (7). Then, they are completed with the accumulation of terms associated with continuity equation adjustment and filtering process, by considering the individual expansion described in appendixes $\mathrm{A}$ and $\mathrm{C}$, in a sequential inspection of the grid meshes. Due to symmetric property of $\mathbf{M}$, only 31 diagonals are saved, reducing considerably the size of the matrix array. For the application of section 4, MUSCAT requires 1.3 megawords of memory to solve the wind field on 72500 grid points and $400 \mathrm{~s}$ of computation time on a HewlettPackard HP-730 workstation.

Finally, the sparse matrix equation is solved with the standard conjugate gradient method. Because the obtained solution of $u, v$, and $w$ satisfies the continuity equation in a least squares sense, and to limit the errors in the vertical velocity component, a refinement of the $w$ estimates is then performed by integrating this equa- tion according to the approach proposed in Chong and Testud (1983) and applied in EODD.

\section{Comparisons of EODD and MUSCAT}

In this section we will compare the relative quality of the MUSCAT and EODD analyses. This evaluation is carried out by first considering real data and then performing numerical tests. These tests based on simulated radar observations help to investigate more precisely the positive effects of MUSCAT in the recovery of the wind components.

\section{a. Real data analysis}

In Chong and Campos (1996), the EODD analysis was applied to the data collected within a squall-line system occurring on 22 February 1992 during TOGA COARE, by the Doppler radars aboard the two NOAA P-3 aircraft (N42 and N43, respectively). Observations from a specific leg simultaneously performed by the two aircraft within the time period 2110-2121 UTC were used to test EODD, by considering, respectively, data from only the $N 43$ track (dual-Doppler scan) and data from both $N 42$ and N43 tracks (quad-Doppler scan). These dual- and quad-Doppler configurations yielded quite comparable results. Because of this, we will focus on comparisons of EODD and MUSCAT applied to dual-Doppler observations only.

To ensure some continuation with the previous work, the same dataset relative to the above-mentioned N43 leg is used in this study. Detailed description of the observations and data preprocessing can readily be found in Chong and Campos (1996). Application of MUSCAT is performed with the same Cartesian grid and interpolation function characteristics as in the EODD analysis, that is, a domain of $73.5 \times 73.5 \times$ $14.5 \mathrm{~km}^{3}$ with a grid resolution of $1.5 \times 1.5 \times 0.5 \mathrm{~km}^{3}$ and a Cressman weighting function with horizontal and vertical influence radii of 2.5 and $1.2 \mathrm{~km}$, respectively. Also, the weights $\mu_{1}$ and $\mu_{2}$ for the continuity equation adjustment and the filtering process of MUSCAT are set to $S^{2}$ ( $S$ is the area of an elementary horizontal grid mesh) and $0.164 S^{4}$, respectively. With these specifications, direct comparison with results presented in Chong and Campos (1996) can be done.

Figures 1 and 2 compare the vertical cross sections of the line-transverse (west-east section) relative flow (with the corresponding radar reflectivity) and of the vertical velocity at $y=33$ and $y=60 \mathrm{~km}$, respectively, obtained from EODD (Figs. 1a and 2a) and MUSCAT (Figs. $1 \mathrm{~b}$ and $2 \mathrm{~b}$ ). Locations of the aircraft that flew at $170-\mathrm{m}$ altitude are also indicated at $x=16 \mathrm{~km}$ and $x$ $=0 \mathrm{~km}$, respectively. The overall airflow structures from both approaches appear similar (see left panels of Figs. 1 and 2), and this mutual consistency substantiates their validity in solving the three wind components from a minimal configuration of dual-Doppler observations. 

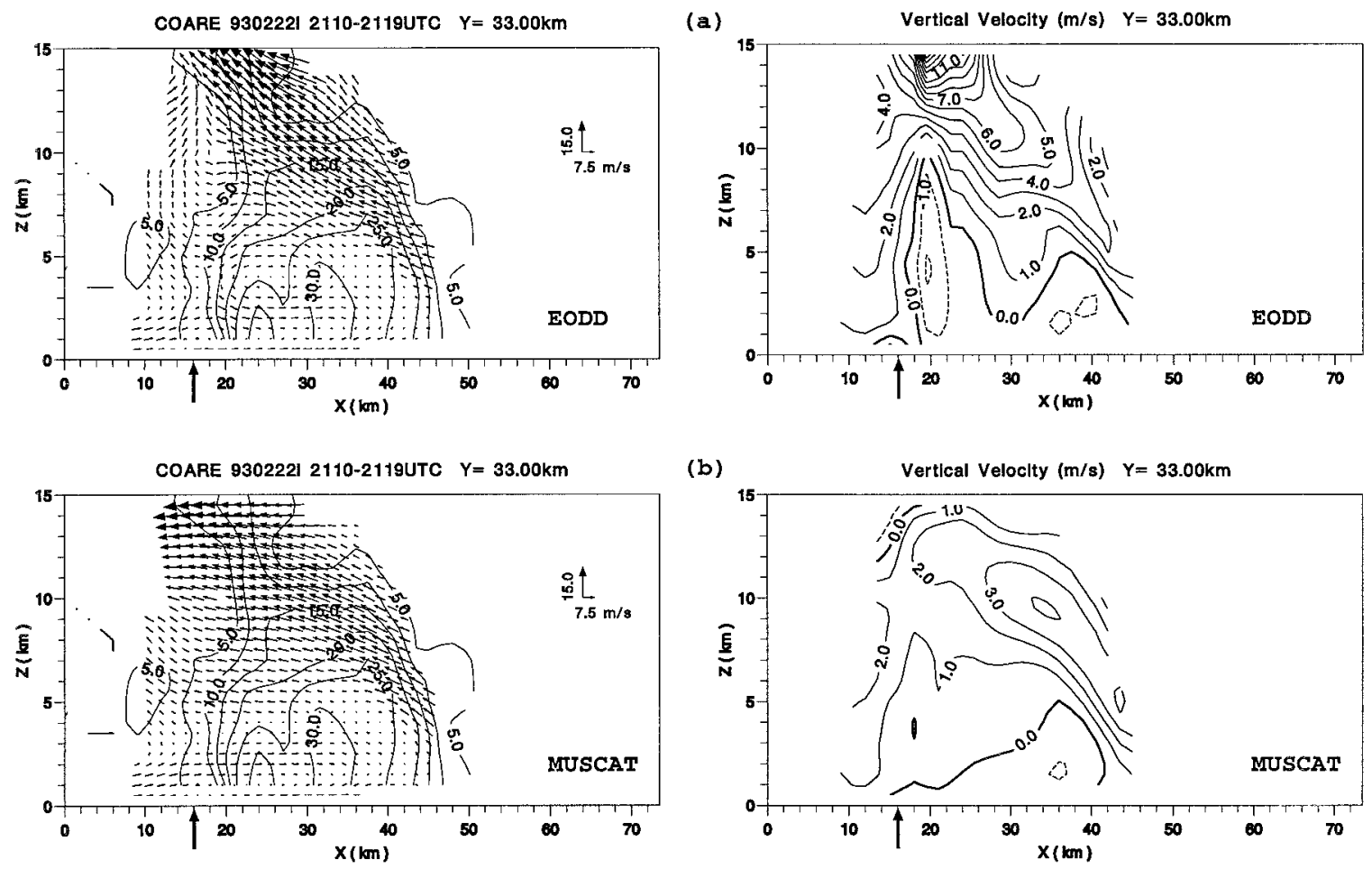

(b)

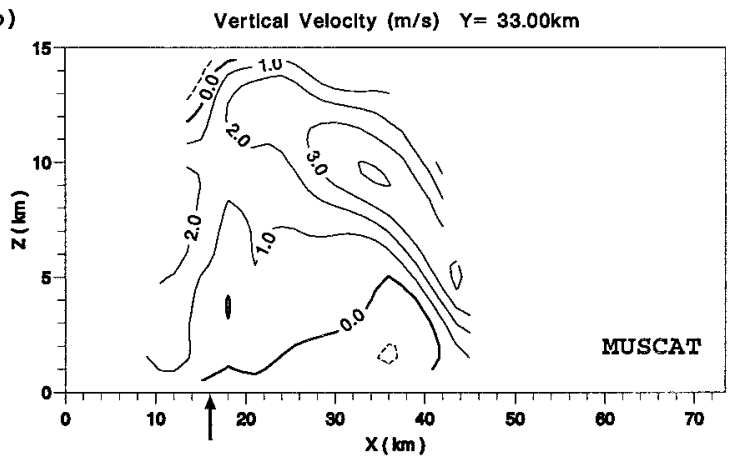

FIG. 1. Vertical cross sections of the relative line-transverse wind and reflectivity (left panels), and vertical velocity (right panels) at $y=$ $33 \mathrm{~km}$. (a) The upper panel corresponds to EODD application, while (b) the lower panel is for MUSCAT results. The vertical arrow below the horizontal axis indicates the aircraft position. The scales for the wind components are also represented.

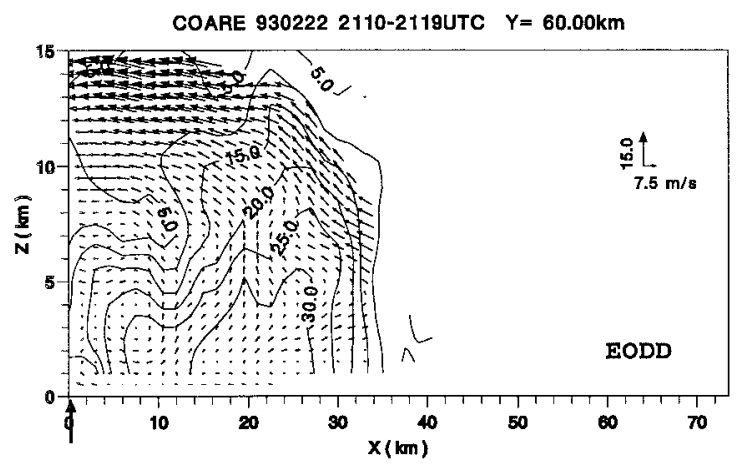

(a)
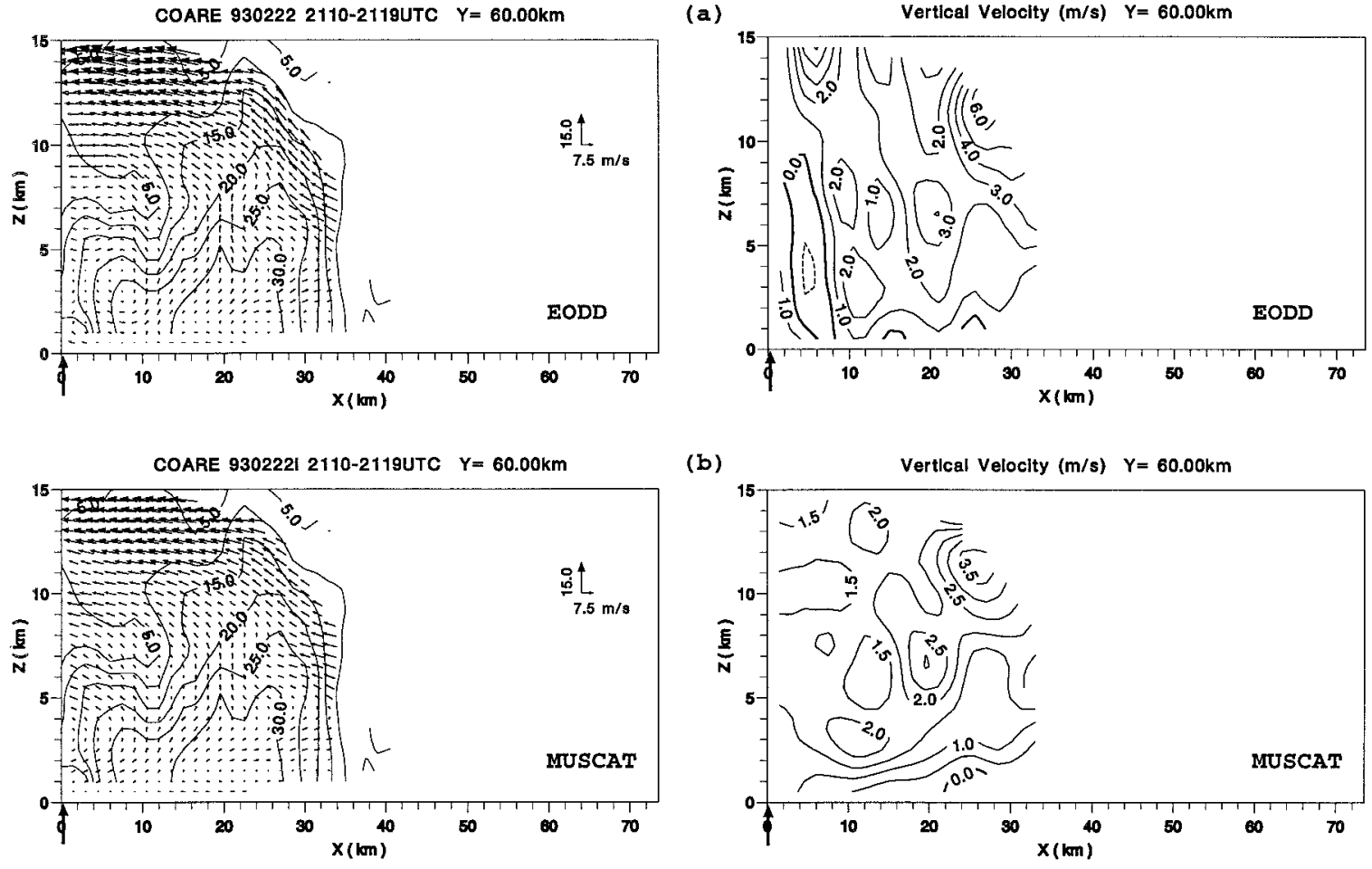

(b)

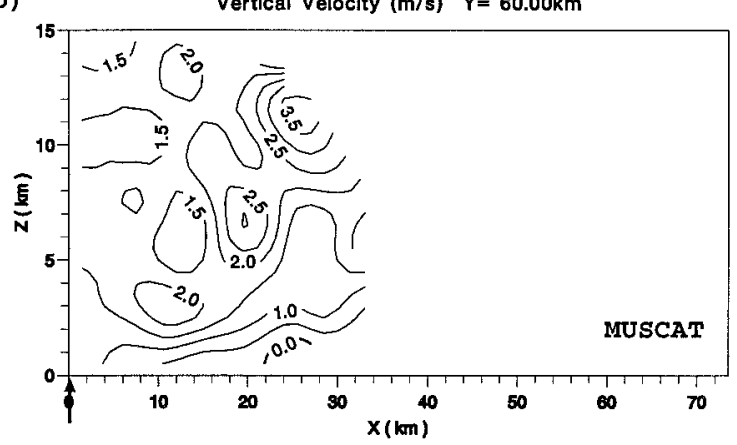

FIG. 2. As in Fig. 1 but at $y=60 \mathrm{~km}$. 
The components from the two analyses are within a few meters per second of each other. However, the detailed features are significantly different, and this can be attributed to improvements due to the simultaneous solution of MUSCAT as opposed to the iterative process of EODD (this will be confirmed in the next section). The differences are noticeable for the cross section at $y=33 \mathrm{~km}$ (Fig. 1), which displays an upper-level updraft ( $>10 \mathrm{~m} \mathrm{~s}^{-1}$ above 13-km altitude) in the EODD analysis (Fig. 1a), while MUSCAT provides a main updraft reaching a maximum of $4 \mathrm{~m} \mathrm{~s}^{-1}$ at $10-\mathrm{km}$ altitude and then decreasing progressively to less than $1 \mathrm{~m} \mathrm{~s}^{-1}$ at top levels (Fig. 1b). Moreover, an area of downward motions less than $-1 \mathrm{~m} \mathrm{~s}^{-1}$ is observed at $x=18-23$ $\mathrm{km}$, beneath the EODD-derived upper-level updraft. There is a similar updraft-downdraft structure in the EODD analysis in Fig. 2, at $x<8 \mathrm{~km}$, which is not revealed in MUSCAT results. In fact, this major discrepancy occurs in regions close to the flight track, where different conclusions about storm mechanisms would result from using the two different approaches. EODD depicts convergent flows composed of the main upward front-to-rear flow (from right to left) opposing a rear-to-front flow (from left to right) at high levels greater than $8 \mathrm{~km}$. This contrasts with the overall upward front-to-rear flow from MUSCAT.

These differences on the line-transverse components are also observed on the line-parallel ones, as shown in
Fig. 3, which compares the $u$ and $v$ components obtained from EODD (Fig. 3a) and MUSCAT (Fig. 3b) at $y=$ $33 \mathrm{~km}$. The associated line-transverse $(\partial u / \partial x)$ and total $(\partial u / \partial x+\partial v / \partial y)$ divergence patterns are shown in Fig. 4. In most respects, MUSCAT provides a smooth structure in wind components and divergence, while EODD can produce strong gradients, especially in regions above the aircraft. A rapid reversal of the components is obtained from the EODD analysis, which contrasts with the gradual variation found in the MUSCAT-derived components. The high level region of strong vertical velocities from EODD (Fig. 1a) clearly results from the convergence zone above 8 -km altitude (Fig. 4a). The existence of such an area of convergence is questionable at storm top, where virtually any numerical or conceptual model would indicate an area of divergence. Numerical simulations of the present observational case (Trier et al. 1996) show a progressive decrease of the upward motions at top levels. This corroborates the MUSCAT results, which show a divergent upper-level flow (Fig. 4b) quite consistent with the detrainment of updraft air toward the rear of the system, as previously documented (see, e.g., Houze and Betts 1981; Chong et al. 1987). At these levels above the aircraft, the contribution of the incorrect vertical velocities from the EODD analysis is maximized, which increases the errors in the determination of the horizontal components during the iterative process.

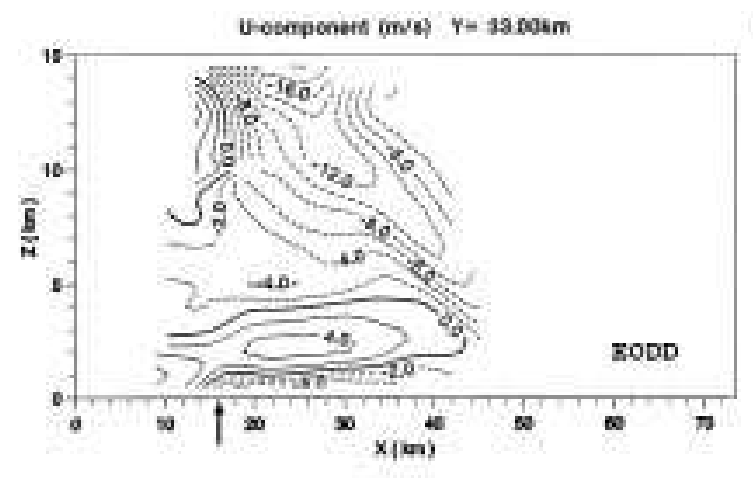

(a)
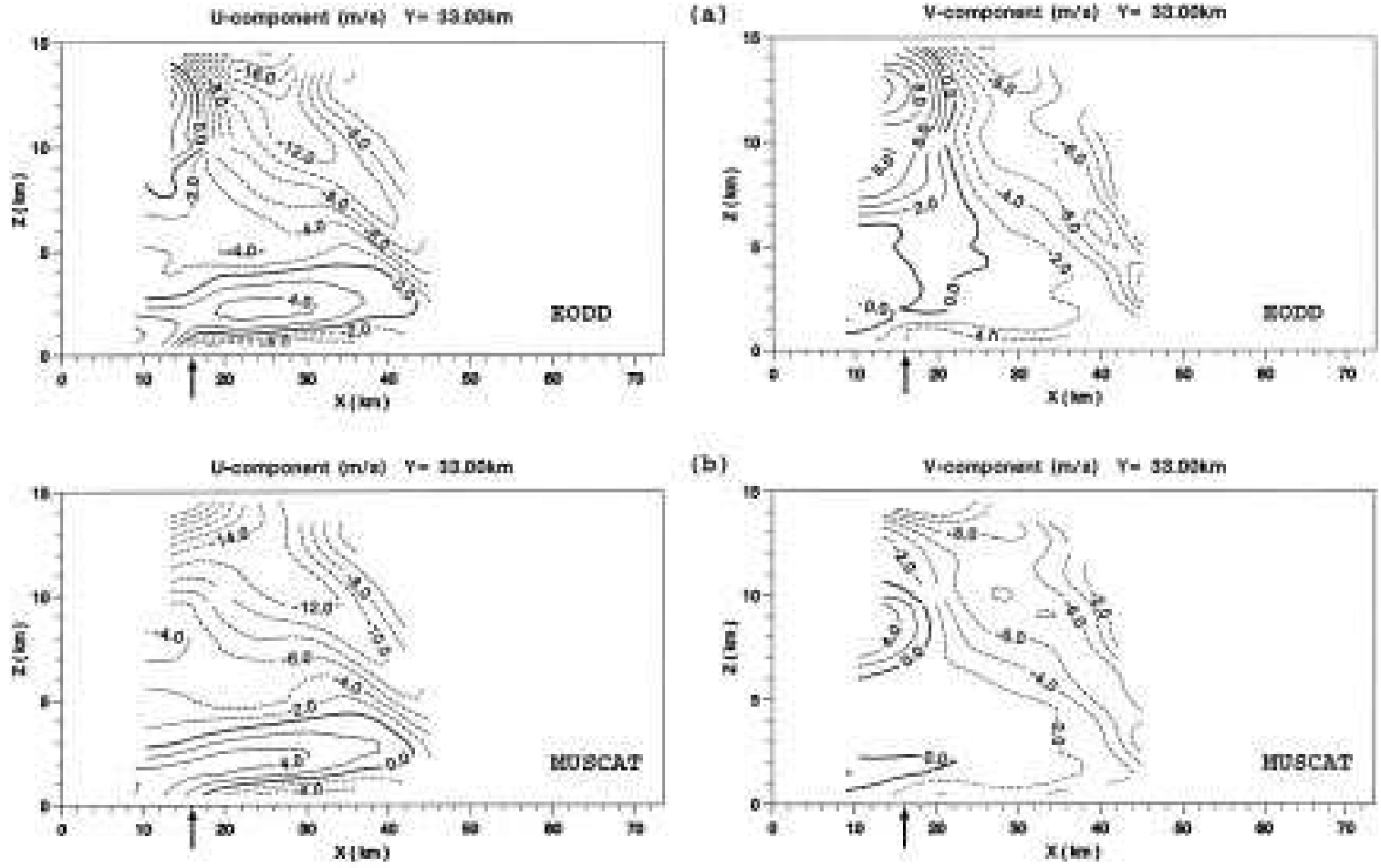

(b)

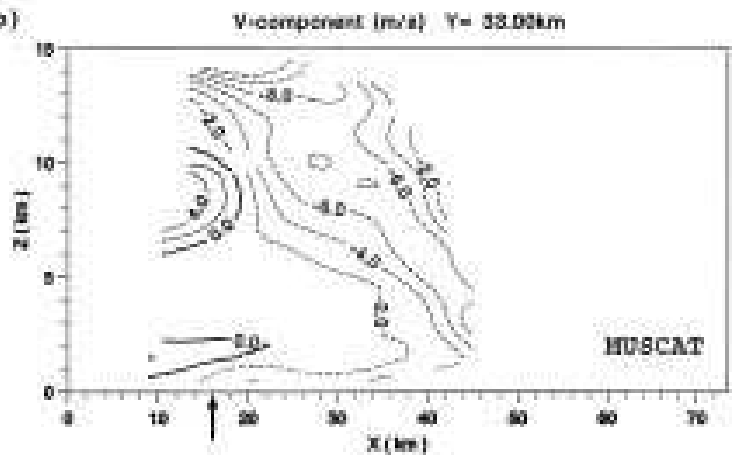

FIG. 3. Vertical cross sections of the line-transverse $u$ (left panels) and line-parallel $v$ (right panels) at $y=33 \mathrm{~km}$. (a) The upper panel results from EODD, while (b) the lower panel is obtained from MUSCAT. The vertical arrow below the horizontal axis indicates the aircraft position. 

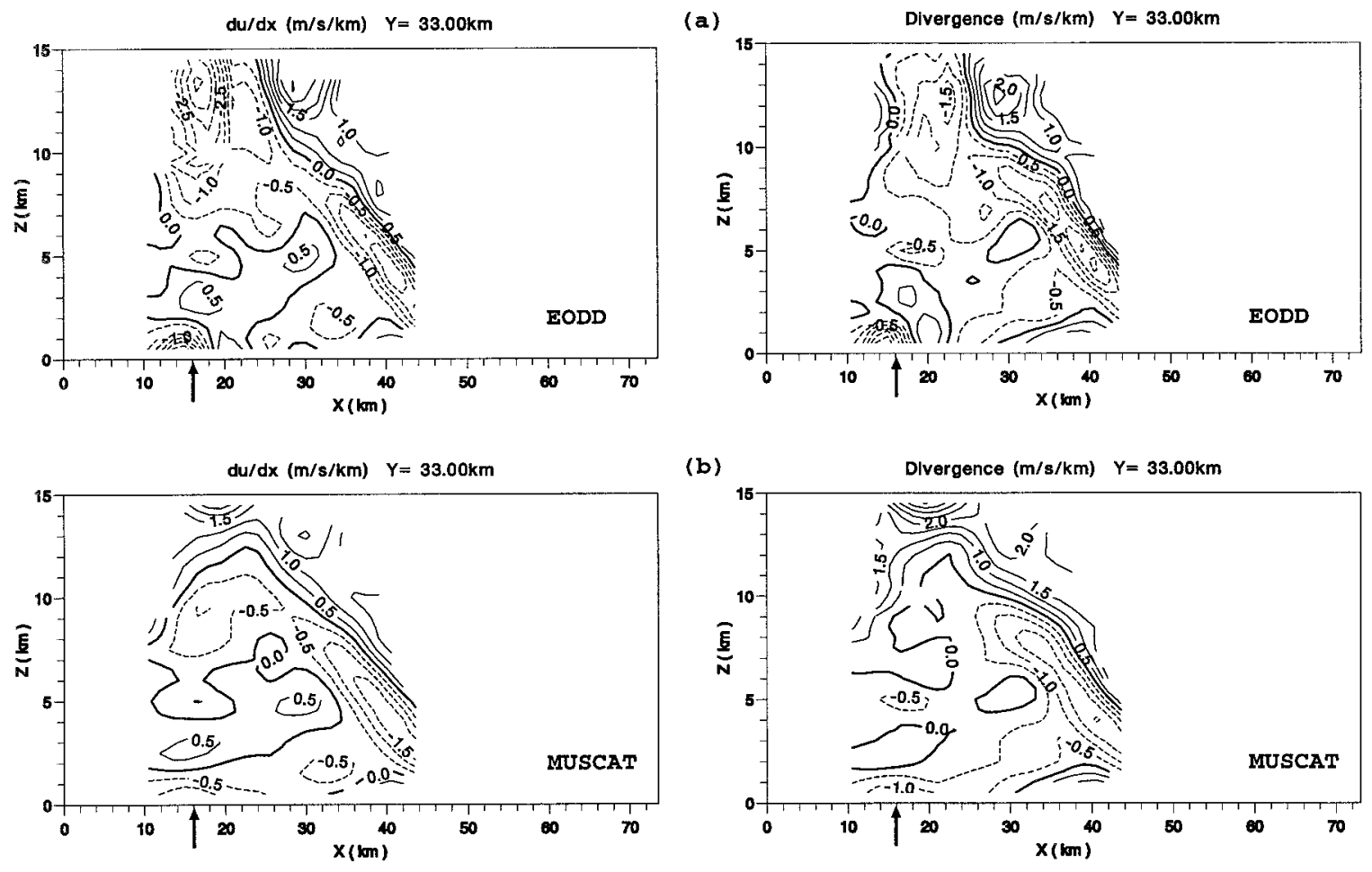

FIG. 4. As in Fig. 3 but for line-transverse $\partial u / \partial x$ and total $\partial u / \partial x+\partial v / \partial y$ divergence.

At low levels, although the $u$ and $v$ components (Fig. 3) depict similar features, there are small-scale variations in the EODD analysis that strongly affect the convergence zone between the deep rear-to-front flow (positive $u$ ) and the shallow downdraft-generated diverging outflow toward the rear of the convective cells (negative $u$ ), as revealed by MUSCAT (Fig. 4b). As discussed in section $2 b$, incorrect winds can explain the occurrence of the doublets of convergence/divergence that can be easily observed on the total divergence field of Fig. 4a and that extend between $x=10 \mathrm{~km}$ and $x=22 \mathrm{~km}$, which is still in regions close to and above the aircraft. Note that in these areas in which EODD fails other dualDoppler approaches will likely fail also.

\section{b. Numerical tests}

Comparison of the application of EODD and MUSCAT to real data has clearly shown noticeable differences in the derived wind structure, particularly in regions above the aircraft track. Expected limitations of EODD were put forward to explain these differences, while previously documented airflow structure within squall lines was suggested to evaluate the consistency of the wind retrieval from MUSCAT. However, this comparison remains qualitative since the reference wind field (exact solution) cannot be observed in nature. An alternative consists of using simulated radar observations and performing a quantitative analysis of the improvements of MUSCAT against EODD. Such an ap- proach was also used by Chong and Testud (1996) in their study of the coplane methodology to synthesize airborne Doppler radar data. We adopt the same procedure to obtain numerical tests close to experimental conditions, that is, we consider the dual-beam sampling from the leg analyzed in the previous section and substitute the observed radial velocities with those derived from an analytic form of the wind components. To simulate the radar statistical error, random errors with an rms value of $1 \mathrm{~m} \mathrm{~s}^{-1}$ are then added to these radial components.

\section{1) Simulated Wind Field}

The two-dimensional wind field used in Chong and Testud (1996) is considered, and it derives from a description of the vertical velocity $w$ in terms of a product of two sine functions defined as

$$
w=A \sin k_{z} z \sin k_{H}\left(r_{H}-a z-b\right),
$$

where $A$ is the amplitude; $k_{H}$ and $k_{z}$ are, respectively, the horizontal and vertical wavenumbers; $r_{H}$ is the horizontal distance; and $a$ and $b$ are parameters defining a linear variation of the phase shift with height. Note that $w=0$ is always verified at the surface and that implicitly $k_{H}$ and $r_{H}$ are colinear. In the vertical plane containing the wave vectors, $r_{H}=a z+b$ defines the line for which $w=0$, as do the parallel lines at a horizontal distance that is a multiple of half the horizontal wavelength. 
Considering the mass continuity equation (3), the horizontal wind $V_{H}$ in the $r_{H}$ direction can be expressed as

$$
\begin{aligned}
V_{H}= & \frac{A}{k_{H}}\left(k_{z} \cos k_{z} z-\kappa \sin k_{z} z\right) \cos k_{H}\left(r_{H}-a z-b\right) \\
& +a A \sin k_{z} z \sin k_{H}\left(r_{H}-a z-b\right) .
\end{aligned}
$$

In this study, the horizontal wave vector was assumed along the $x$ direction (i.e., $u=V_{H}$ and $v=0$ ), and horizontal and vertical wavelengths were equal to 40 and $24 \mathrm{~km}$, respectively. Amplitude $A$ was set to $10 \mathrm{~m}$ $\mathrm{s}^{-1}$ and $\kappa$, the inverse of the scale height of the air density decrease, was assumed to be equal to 0.1 . For simplicity, the analysis domain was limited to $48 \mathrm{~km}$ in the $x$ direction since no observation was available beyond that limit and to $12 \mathrm{~km}$ in the vertical where $w$ vanishes. Parameters $a$ and $b$ were chosen to be equal to $-0.7 \mathrm{~km}^{-1}$ and $17 \mathrm{~km}$, respectively. The corresponding $y$-independent two-dimensional wind field and the associated $u$ and $w$ components are shown in Figs. 5a$c$, respectively. With the above specifications, the simulated airflow consists of two opposite vortex circulations sloping to the left with an angle of $35^{\circ}$ and centered at $x=13.8$ and $33.8 \mathrm{~km}$, and $z=4.6 \mathrm{~km}$. Upward motions are in the central part of the domain and reach $10 \mathrm{~m} \mathrm{~s}^{-1}$, as do the downdrafts on either side. Horizontal velocities range from 0 to $\pm 18 \mathrm{~m} \mathrm{~s}^{-1}$.

\section{2) Results}

Figures $6 \mathrm{a}$ and $6 \mathrm{~b}$ visualize the horizontal flow at 5$\mathrm{km}$ altitude, as deduced from the application of MUSCAT and EODD to the simulated data. The aircraft track is also reported. While MUSCAT provides a horizontal flow quite similar to the simulated one (not shown), strong perturbations occur near the flight track, especially in its northern portion, when EODD is considered. Reversal of the flow components as discussed above can be easily identified: a southwest-oriented flow is generated, which contrasts with the original west-east flow and substantially modifies the convergence patterns. Although the differences between MUSCAT and EODD are very localized, this example highlights the real improvements of MUSCAT to synthesize the horizontal components.

To investigate further the compared performances of MUSCAT against EODD, Figs. 7 and 8 present vertical sections of the airflow and the corresponding $u, v$, and $w$ components from both approaches at $y=60 \mathrm{~km}$, where differences are maximized. Again, the aircraft location at $x=0 \mathrm{~km}$ is materialized. It can be seen that MUSCAT correctly reproduces the flow features (compare Figs. 5 and 7) with imperceptible discrepancy. Only very small perturbations in the $v$ components (assumed to be zero) are observed. On the contrary, results from EODD (Fig. 8) depict major differences with the actual wind field, which concern all levels in regions close to the aircraft $(x<10 \mathrm{~km})$. Note the large errors in the $v$
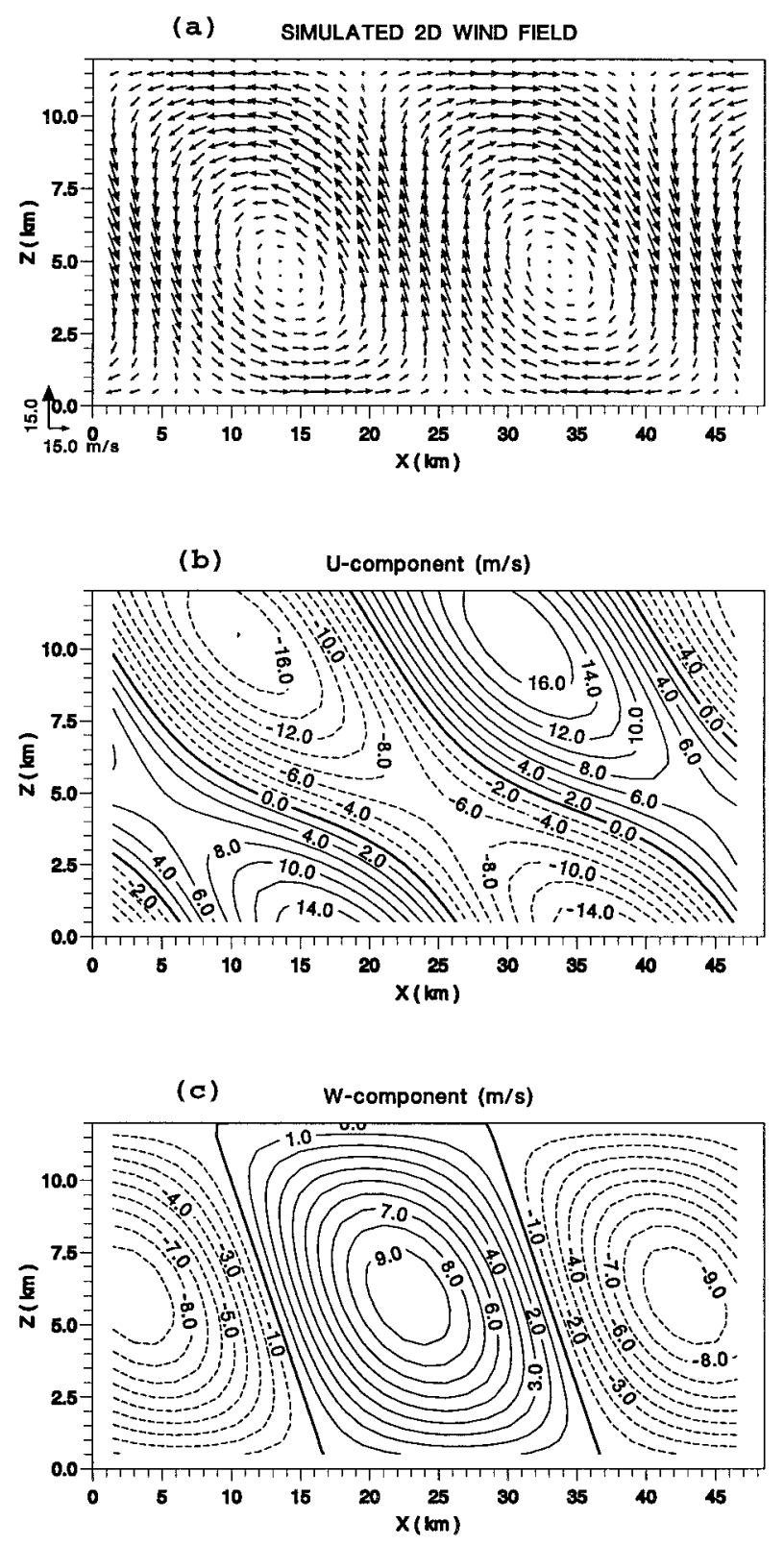

FIG. 5. Vertical representation of the 2D wind field used to simulate Doppler radar observations: (a) vector representation, (b) horizontal component, and (c) vertical velocity.

component (Fig. 8c), which are also associated with large errors in the $u$ and $w$ components. In particular, artificial negative $u$ and $v$ instead of positive $u$ and nonexistent $v$ are generated, modifying dramatically the vortex circulation. A consequence is the complete reorganization of the vertical motions associated with unrealistic convergence/divergence patterns attached to such flow. An updraft core at low levels is observed, which tends to squeeze the downdraft structure to the left side of the domain and changes the actual left-hand sloping downdraft to a right-hand orientation. In terms of negative effects of EODD when an inadequate so- 
(a)

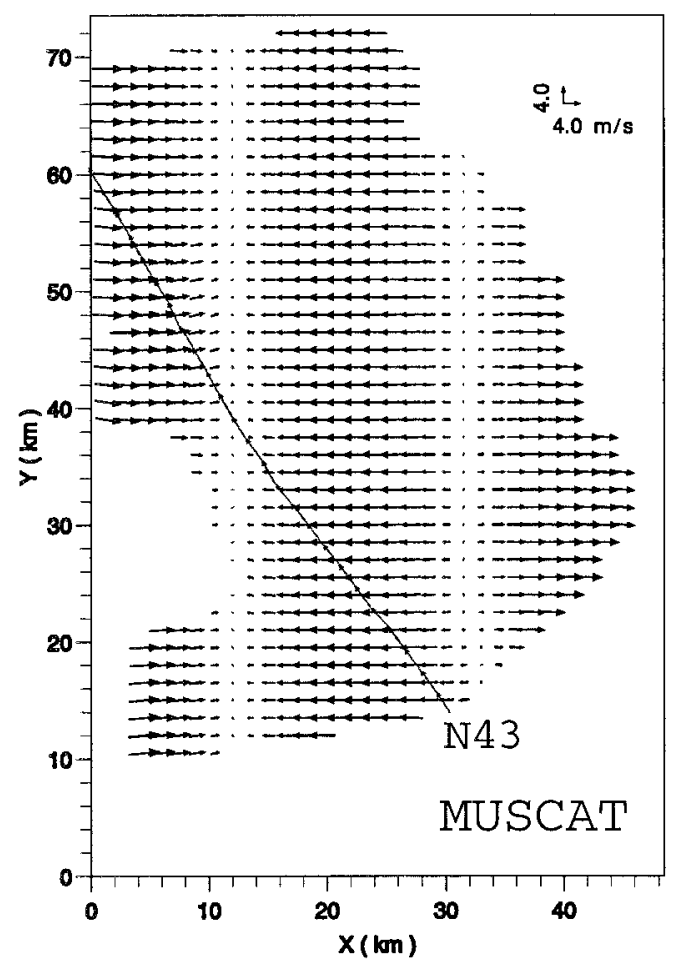

(b)

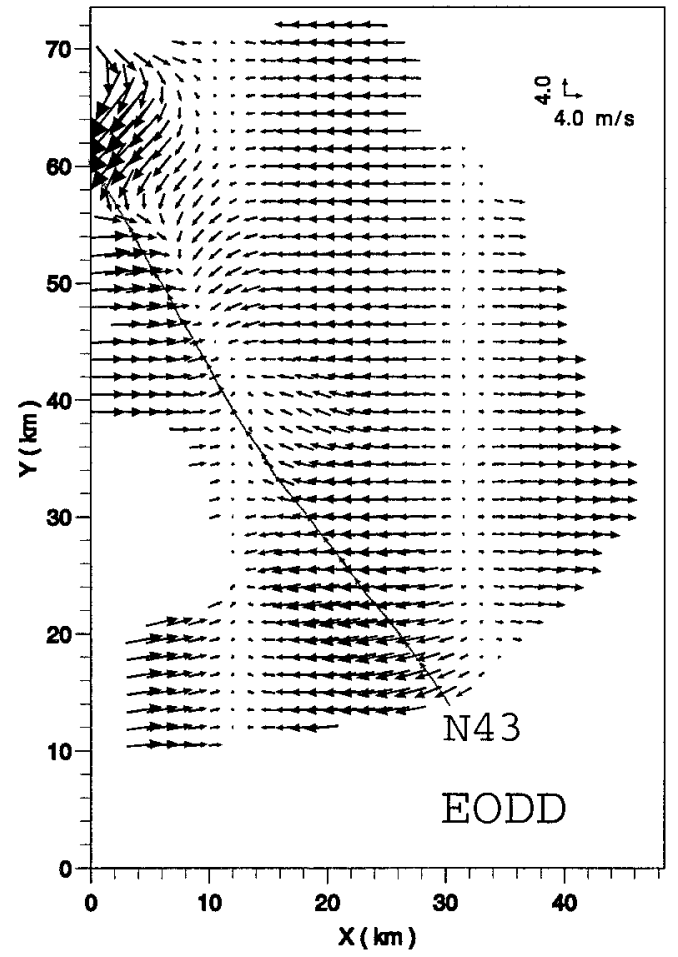

FIG. 6. Retrieved horizontal flow at 5-km altitude from (a) MUSCAT and (b) EODD, as applied to the simulated data. Oblique line refers to the flight track.

lution is obtained, a parallel one can be readily established with the results shown in Figs. 1a and 3a for real data, which supports the previous hypothesized reason for failure of EODD and improvements of MUSCAT. Considering the limitation to $45^{\circ}$ elevation angle from the radar position in Figs. 8a-d yields a good compromise for dual-Doppler analyses, but these work well at longer ranges. At shorter ranges, the finite interpolation function smears the data, resulting in increasing errors, particularly for ranges less than about $5 \mathrm{~km}$ and in locations where the iterative corrections from EODD cannot adequately function.

MUSCAT is also more accurate than EODD in regions where the latter can provide reliable wind field. An example is given in Figs. 9 and 10, which show the cross sections of the wind components at $y=33 \mathrm{~km}$. Overall, the two methods yield results quite comparable with the actual wind structure shown in Fig. 5. However, small-scale variations are associated with EODD-derived components (Fig. 10) that denote not only residual errors again above the flight track but also in distant regions where EODD is expected to work well (see the vertical velocity pattern at $x>35 \mathrm{~km}$ ). This is probably related to the iterative resolution of horizontal and vertical components, which is not so stable as the simultaneous determination of MUSCAT. Comparison of Figs. 8 and 10 seems to indicate that the problem with
EODD, in Fig. 8, is presumably that the data are unbounded on the left, whereas, in Fig. 10, there are data on both sides of the flight track. Another cause is the contribution of the vertical motions, which is more important. We found that taking another value for $b$ in (13), such that the vertical velocity fluctuates about zero near $x=0 \mathrm{~km}$, significantly reduced the biases shown in Fig. 8.

As a summary, Fig. 11 gives the statistics of the errors that can be evaluated as the differences between retrieved and simulated components. At each level, the mean and standard deviation of these differences for $u$, $v$, and $w$ are displayed. Note that these errors do not fully represent the errors in an actual analysis since other sources of errors are not considered in this study, such as those associated with aircraft positioning or radar beam pointing angles (e.g., Hildebrand and Mueller 1985). The comparison of the various profiles of the mean differences and associated standard deviations shows the effective performance of MUSCAT, which provides the most stable results. [We verified that imposing horizontal and vertical wavelengths of 30 and $30 \mathrm{~km}$ for the wind field (i.e., reducing the horizontal extension of the updraft-downdraft structure and prescribing a nonzero vertical velocity at the top) did not modify these results.] Errors in the three components are quite comparable in magnitude: mean values are less 
(a) RETRIEVED FIELD $Y=60.00 \mathrm{~km}$

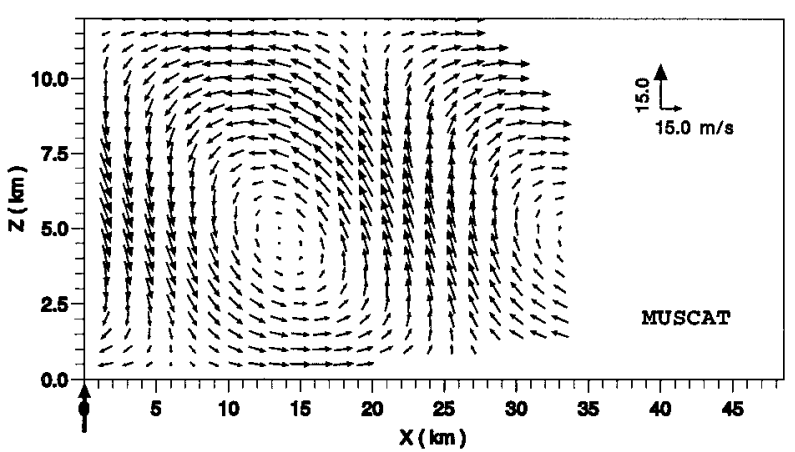

(b) U-component $(\mathrm{m} / \mathrm{s}) \quad \mathrm{Y}=60.00 \mathrm{~km}$

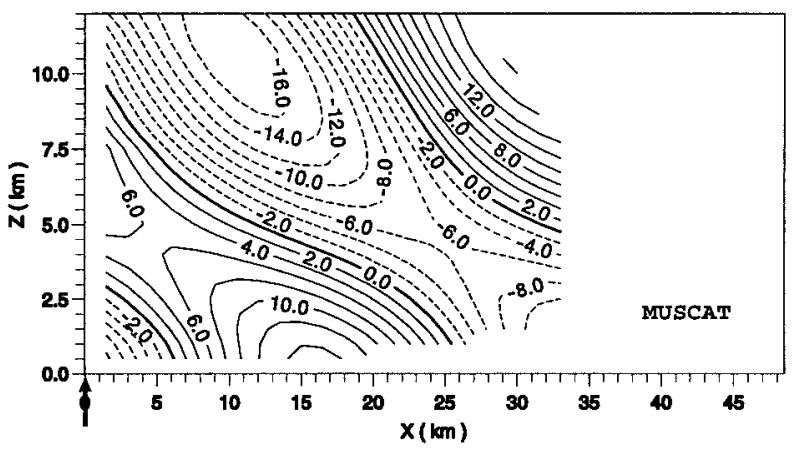

(c) V-component $(\mathrm{m} / \mathrm{s}) \quad Y=60.00 \mathrm{~km}$

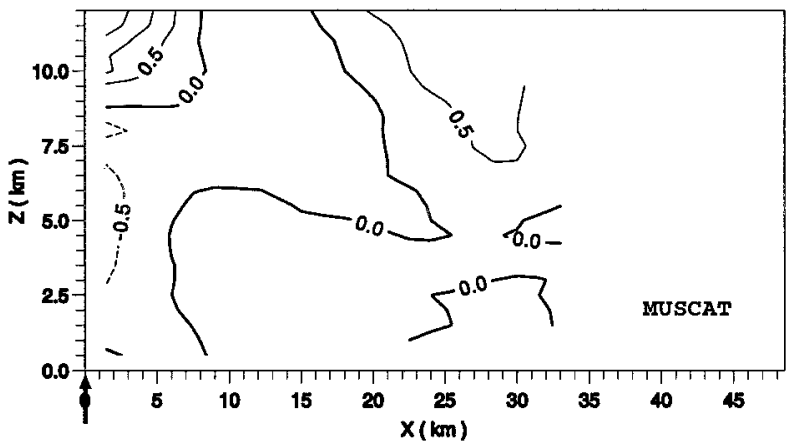

(d) W-component $(\mathrm{m} / \mathrm{s}) \quad Y=60.00 \mathrm{~km}$

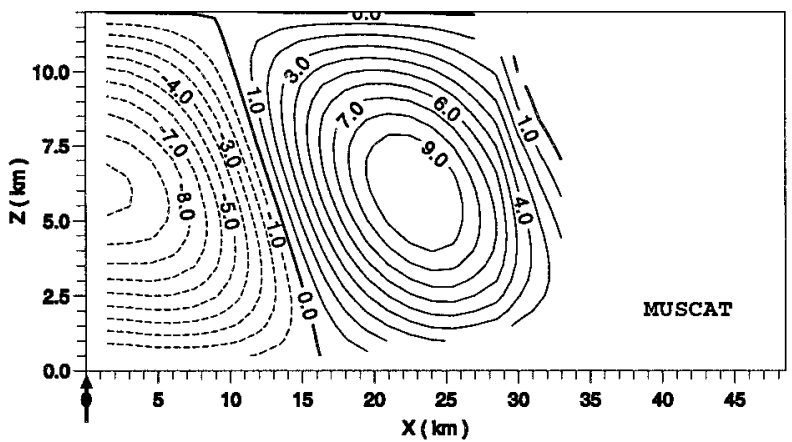

FIG. 7. MUSCAT-derived vertical wind structure at $y=60 \mathrm{~km}$ : (a) vector representation, (b) $u$ component, (c) $v$ component, and (d) $w$ vertical velocity. Aircraft position is identified by the vertical arrow below the horizontal axis. (a) RETRIEVED FIELD $Y=60.00 \mathrm{~km}$

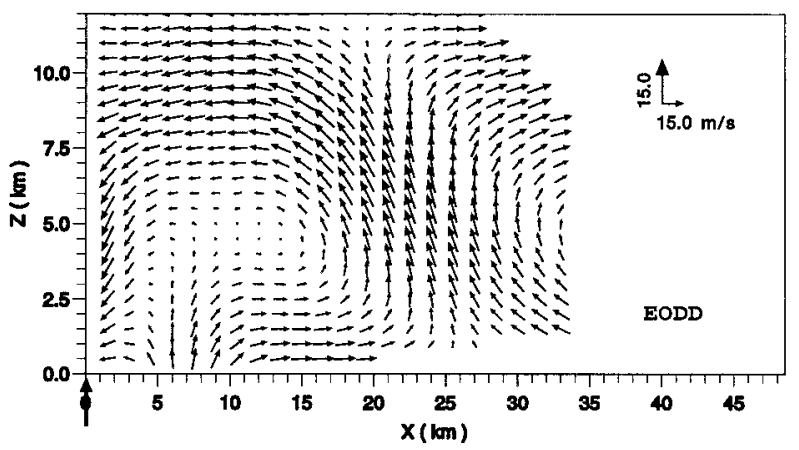

(b) U-component $(\mathrm{m} / \mathrm{s}) \quad Y=60.00 \mathrm{~km}$

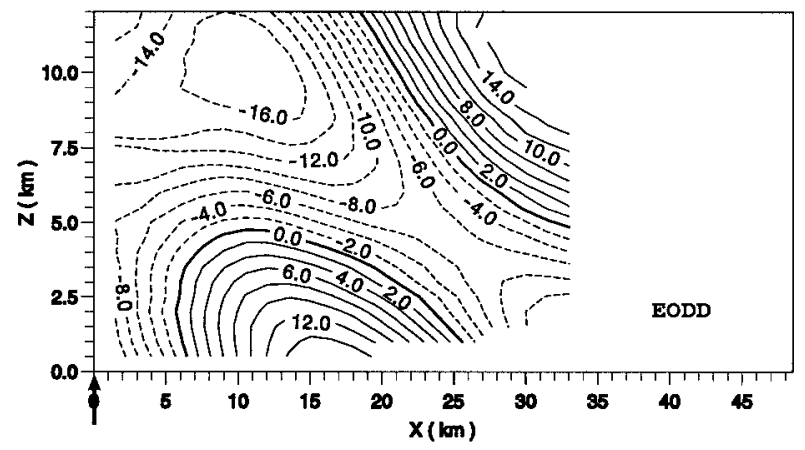

(c) V-component $(\mathrm{m} / \mathrm{s}) \quad Y=60.00 \mathrm{~km}$

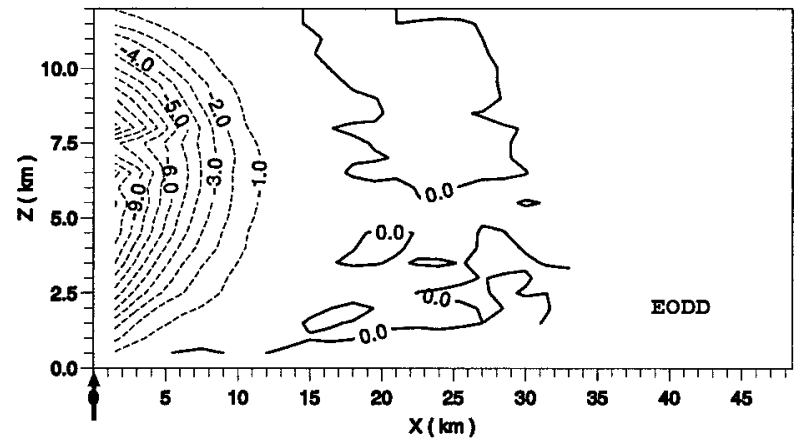

(d) W-component $(\mathrm{m} / \mathrm{s}) \quad Y=60.00 \mathrm{~km}$

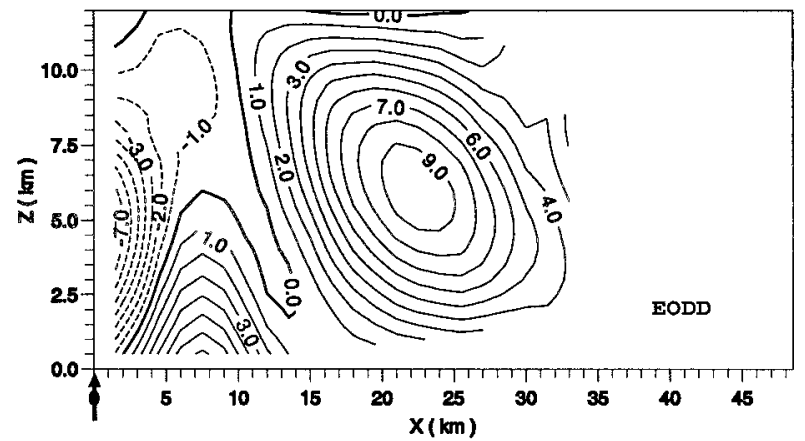

FIG. 8. As in Fig. 7 but for EODD application. 
(a) U-component $(\mathrm{m} / \mathrm{s}) \quad \mathrm{Y}=33.00 \mathrm{~km}$

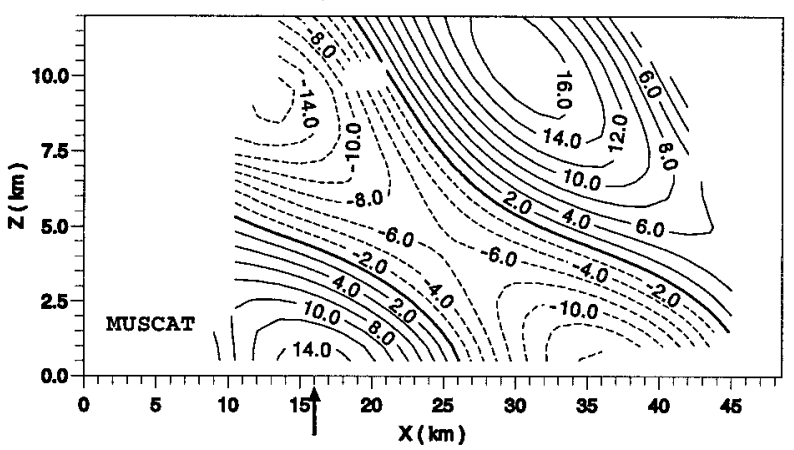

(b) V-component $(\mathrm{m} / \mathrm{s}) \quad \mathrm{Y}=33.00 \mathrm{~km}$

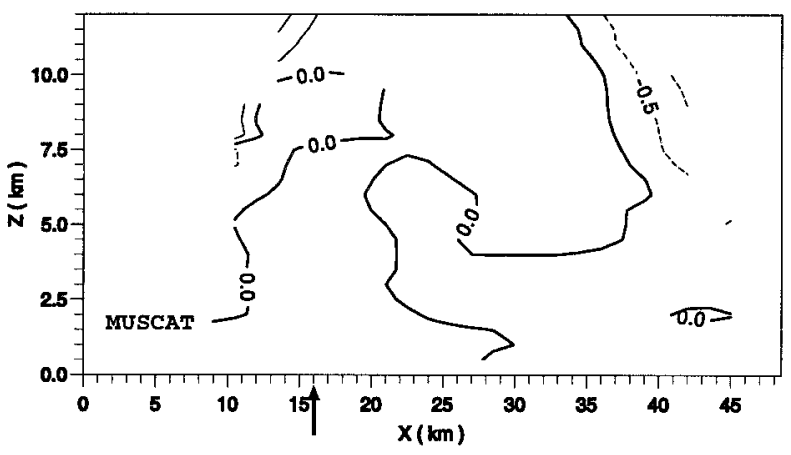

(c) W-component $(\mathrm{m} / \mathrm{s}) \quad \mathrm{Y}=33.00 \mathrm{~km}$

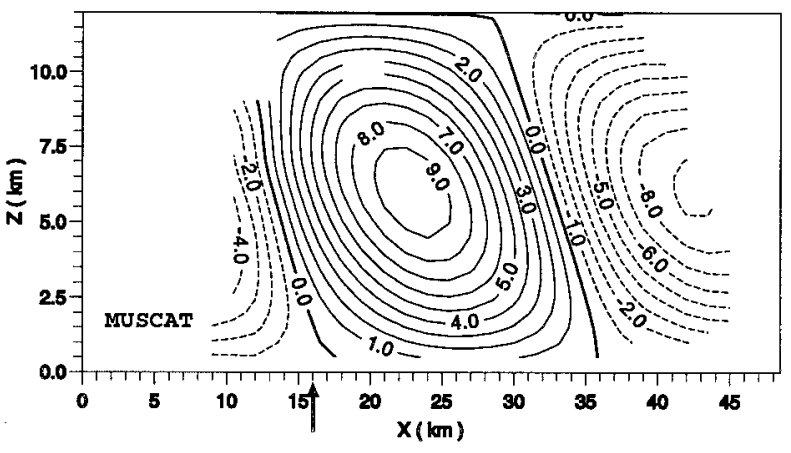

FIG. 9. Contours of (a) $u$, (b) $v$, and (c) $w$ in the vertical plane at $y=33 \mathrm{~km}$, as obtained from MUSCAT. Aircraft position is identified by the vertical arrow below the horizontal axis.

than $0.2 \mathrm{~m} \mathrm{~s}^{-1}$, while standard deviations remain within $0.7 \mathrm{~m} \mathrm{~s}^{-1}$. On the other hand, these errors are significantly increased when EODD is used. Mean errors (in absolute value) and standard deviations are closely related since they have similar height variations whatever the component may be. These errors that are mainly due to the unstable solutions of the horizontal components can be considered as bias errors. Both $u$ and $v$ biases depict similar variations with pronounced extrema around the 8-km level: the mean biases reach -1.0 and $-0.6 \mathrm{~m} \mathrm{~s}^{-1}$ with standard deviations of 3.4 and $2.0 \mathrm{~m}$ $\mathrm{s}^{-1}$, respectively. Errors in the vertical velocities can reach a mean value of $1.0 \mathrm{~m} \mathrm{~s}^{-1}$ and a standard deviation of $2.7 \mathrm{~m} \mathrm{~s}^{-1}$ around $5-\mathrm{km}$ altitude. (a) U-component $(\mathrm{m} / \mathrm{s}) \quad Y=33.00 \mathrm{~km}$

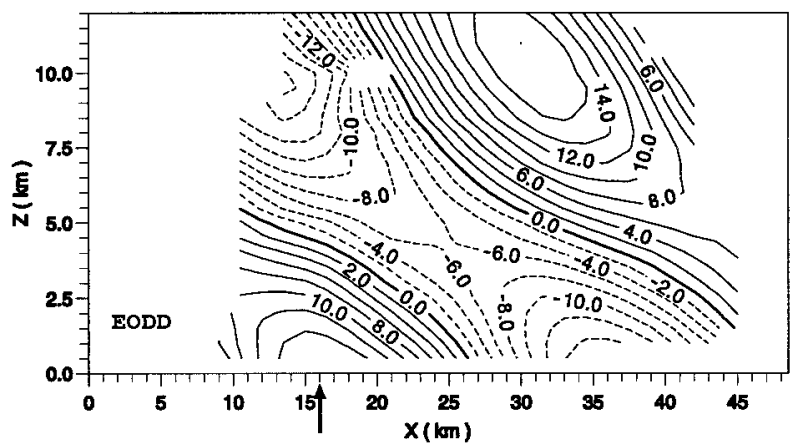

(b) V-component $(\mathrm{m} / \mathrm{s}) \mathrm{Y}=33.00 \mathrm{~km}$

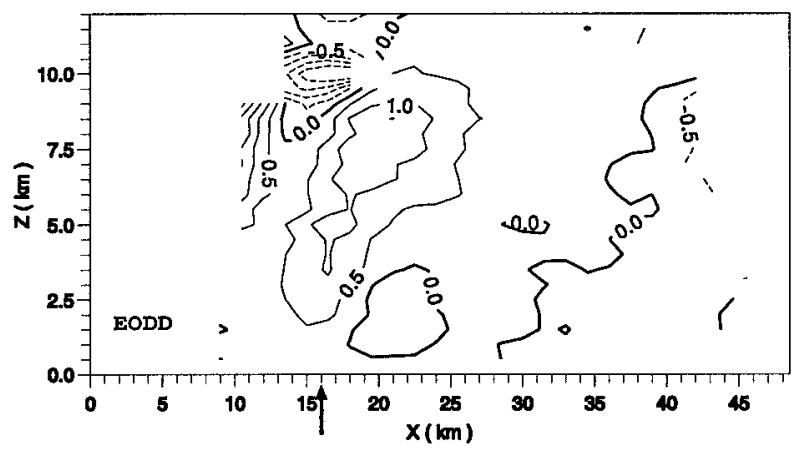

(c) W-component (m/s) Y=33.00km

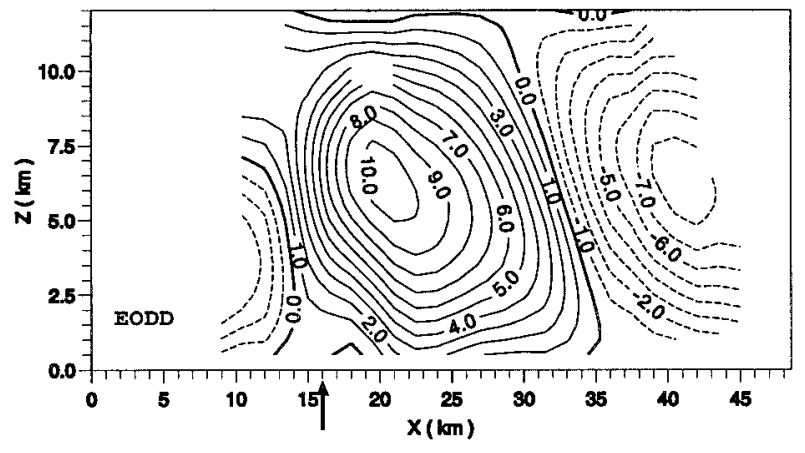

FIG. 10. As in Fig. 9 but for EODD.

\section{Conclusions}

This paper has examined a new approach to synthesizing the three-dimensional wind field from dual-Doppler radar observations. The multiple-Doppler synthesis and continuity adjustment technique has been developed to solve, more efficiently, the major problems arising from any Cartesian dual-Doppler analysis that is based on an iterative resolution with the physical mass continuity equation. MUSCAT is designed to improve upon the extended overdetermined dual-Doppler (EODD) analysis, as proposed by Chong and Campos (1996), which has shown a real ability to produce highly reliable flow structure in regions where the traditional techniques fail. EODD was primarily designed to overcome the severe geometrical limitations of the data analysis 
MUSCAT
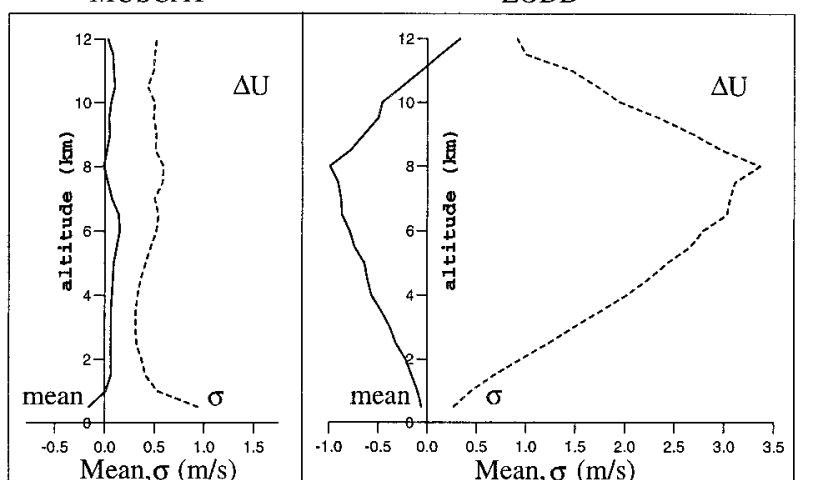

$\left.{ }^{12}\right]$

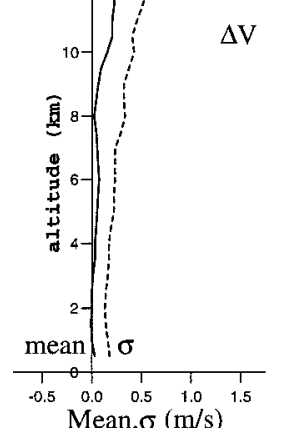

$\mathrm{V}$

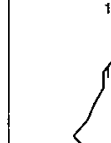

127

Mean, $\sigma(\mathrm{m} / \mathrm{s})$
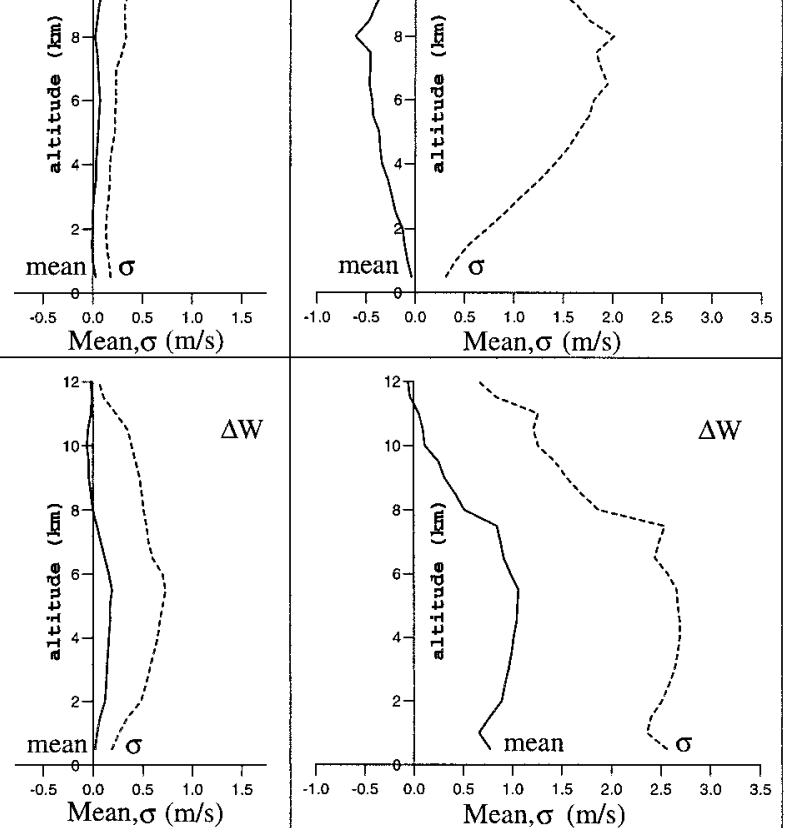

FIG. 11. Height profiles of the mean and standard deviation of the differences between synthesized and simulated $u, v, w$ components (from top to bottom). Left panels refer to MUSCAT-simulated differences, while right panels are for EODD-simulated differences.

of airborne Doppler radars capable of fore-aft scanning in either a dual-Doppler or a quad-Doppler (combining coordinated and parallel flight tracks of a two-plane mission) configuration. However, the EODD-resolved wind field can contain residual errors since the three wind components are not obtained simultaneously.

MUSCAT provides a simultaneous resolution of the three wind components and satisfies both the minimal dual-equation system and the continuity equation. The EODD technique considered the continuity equation as a constraint for the least squares fit of the horizontal components to grid-interpolated Doppler data in individual planes and also constrained their second derivatives in order to limit the small-scale variations. This variational formalism has been fully retained in MUSCAT; only the associated minimization problem has been extended to the vertical wind component. Some accommodations have been necessary, however, to account for the three-dimensional continuity equation in a plane-to-plane analysis or to extend the filtering of small-scale variations to this component. A detailed inspection of the filtering terms has been performed to obtain an isotropic response of the process. Refinements have also concerned the data fit in the sense that data interpolation has been included.

To analyze the improvements of MUSCAT over EODD, applications to airborne Doppler observations of a squall line in TOGA COARE and to simulated radar observations were carried out. The real data clearly showed noticeable differences in the derived wind structure. Although the components were within a few meters per second of each other, some aspects of the EODD synthesis were scientifically unrealistic, whereas MUSCAT corrected these problems. Examples in this paper illustrate residual errors in EODD-resolved winds that produced strong and inconsistent gradients associated with a rapid reversal of the components. MUSCAT provides a more regular airflow, which is consistent with previously documented flow patterns. Results from the numerical tests using simulated data emphasize the real improvements of MUSCAT since it was able to reproduce the characteristics of the simulated flow structure with imperceptible discrepancy. MUSCAT provided the most stable results in the sense that errors in the three wind components were small and comparable. Finally, this study has shown that a mathematically exact solution necessitates a simultaneous resolution of the wind components and has also revealed the importance of numerical tests to investigate more precisely the performances or failures of data analysis techniques that could not evidently be detected from observed data.

\section{APPENDIX A}

\section{Discrete Form of the Continuity Equation Adjustment}

We consider a three-dimensional grid of dimension $m \times n$ in the horizontal ( $m$ grid points along $x$ axis, $n$ along $y$ axis) and $p$ in the vertical. To simplify the notations and to get a tractable form of the variational problem, horizontal two-dimensional grid points $(i, j)$ are renumbered with a single one-dimensional index $I$ running from 1 to $m n$ and defined as $I=(j-1) m+$ $i$ for $i=1, m$ and $j=1, n$. Subscript $k$ will define the level index. Using off-centered finite differences, term B $^{\prime}$ [Eq. (9)] can be expressed at the center of each elementary grid box $I$ defined by grid points $(I, I+1$, $I+m, I+m+1)$ at levels $k$ and $k-1$, as 


$$
\begin{aligned}
\mathrm{B}_{I k}^{\prime}=\frac{\mu_{1}}{16}\{[ & \left(-u_{I}+u_{I+1}-u_{I+m}+u_{I+m+1}\right)_{k} \\
& \left.+\left(-u_{I}+u_{I+1}-u_{I+m}+u_{I+m+1}\right)_{k-1}\right] / L \\
+ & {\left[\left(-v_{I}-v_{I+1}+v_{I+m}+v_{I+m+1}\right)_{k}\right.} \\
+ & \left.\left(-v_{I}-v_{I+1}+v_{I+m}+v_{I+m+1}\right)_{k-1}\right] / L \\
+ & {\left[2 /\left(\rho_{k}+\rho_{k-1}\right)\right] } \\
& \times\left[\rho_{k}\left(w_{I}+w_{I+1}+w_{I+m}+w_{I+m+1}\right)_{k}\right. \\
& \quad-\rho_{k-1}\left(w_{I}+w_{I+1}+w_{I+m}\right. \\
\quad & \left.\left.\left.\quad w_{I+m+1}\right)_{k-1}\right] / l\right\}^{2},
\end{aligned}
$$

where $L$ and $l$ are the horizontal and vertical grid resolution, respectively.

With this scheme and in the context of a plane-toplane analysis, wind components on either level $k$ or $k$ -1 must be specified. These are upper or lower boundary conditions (especially for the vertical velocities) for the implicit integration of the continuity equation. For example, let us consider an upward plane-to-plane synthesis of the wind field. Wind components obtained at the lower plane in the preceding plane-to-plane analysis serve as input data for the variational problem. However, assumptions should be made when the lower plane data do not exist, in particular when the surface level is involved. In this case, the vertical velocity is fixed to zero, while the horizontal wind components are assumed to be equal to those at the considered level. A similar description at the top level can be used for a downward analysis. A more compact expression for $\mathrm{B}^{\prime}$, which applies to both upward and downward approaches, is

$$
\begin{aligned}
\mathrm{B}_{I}^{\prime}=[ & a\left(-u_{I}+u_{I+1}-u_{I+m}+u_{I+m+1}\right) \\
& +a\left(-v_{I}-v_{I+1}+v_{I+m}+v_{I+m+1}\right) \\
& \left.+b\left(w_{I}+w_{I+1}+w_{I+m}+w_{I+m+1}\right)-X_{I}\right]^{2},
\end{aligned}
$$

where coefficients $a$ and $b$ can be readily found by comparing (A1) and (A2). Term $-X_{I}$ combines all terms involving input wind components, the subscript $I$ is associated with the grid box number. Note that $a$ should be doubled when starting the process.

\section{APPENDIX B}

\section{Forced Isotropic Response of a Low-Pass Filter}

We examine the response of the filter included in functional $F^{\prime}(u, v, w)$ [Eq. (4)] associated with the variational formulation of MUSCAT. This can be predicted by considering the Euler equation of $F^{\prime}$ successively for variables $u, v$, and $w$. The Euler equations are obtained according to the approach presented by Sasaki (1970). If we consider a filtering process that minimizes the second derivatives of the wind components according to the differential operator

$$
J_{2}=\left(\partial^{2} / \partial x^{2}\right)^{2}+2\left(\partial^{2} / \partial x \partial y\right)^{2}+\left(\partial^{2} / \partial y^{2}\right)^{2},
$$

functional $F_{u}^{\prime}$ for the specific variable $u$ (assuming $v$ and $w$ are known variables) can be rewritten as

$$
\begin{gathered}
F_{u}^{\prime}=\int\left[\left(u-u_{0}\right)^{2}+\mu_{1}\left(\partial u / \partial x-\partial u_{0} / \partial x\right)^{2}\right. \\
\left.+\mu_{2} J_{2}(u)\right] d x d y
\end{gathered}
$$

where $u_{0}$ defines observations including $v$ and $w$. Neglecting $\mu_{1}$, the associated Euler equation is given by

$$
\left(u-u_{0}\right)+\mu_{2}\left(\frac{\partial^{4}}{\partial x^{4}}+\frac{\partial^{4}}{\partial y^{4}}+2 \frac{\partial^{4}}{\partial x^{2} \partial y^{2}}\right) u=0,
$$

which transforms in Fourier's space into

$$
\left(u-u_{0}\right)+\mu_{2}\left(k_{x}^{2}+k_{y}^{2}\right)^{2} u=0 .
$$

The response of the filter, given by the ratio $u / u_{0}$, is

$$
u / u_{0}=\frac{1}{1+\mu_{2}|\mathbf{k}|^{4}},
$$

where $\mathbf{k}=\left(k_{x}, k_{y}\right)$ defines the wave vector in the $x-y$ plane. Equation (B5) specifies an isotropic low-pass filter.

In the presence of the $\mu_{1}$ term (this is the basic principle of MUSCAT, which considers the continuity equation to be resolved), the Euler equation associated with (B2) becomes

$$
\left(1-\mu_{1} \frac{\partial^{2}}{\partial x^{2}}\right)\left(u-u_{0}\right)+\mu_{2}\left(\frac{\partial^{4}}{\partial x^{4}}+\frac{\partial^{4}}{\partial y^{4}}+2 \frac{\partial^{4}}{\partial x^{2} \partial y^{2}}\right)=0,
$$

which yields a nonisotropic transfer function defined by

$$
\frac{u}{u_{0}}=\frac{1+\mu_{1} k_{x}^{2}}{1+\mu_{1} k_{x}^{2}+\mu_{2}|\mathbf{k}|^{4}},
$$

To obtain an isotropic response, that is, to recover expression (B3), the second term on the left-hand side of (B6) should be multiplied by $\left(1-\mu_{1} \partial^{2} / \partial x^{2}\right)$. This implies additional terms to $J_{2}(u)$ that can be readily found to be

$$
J_{2}^{\prime}(u)=\mu_{1}\left[\left(\partial^{3} u / \partial x^{3}\right)^{2}+2\left(\partial^{3} u / \partial x^{2} \partial y\right)^{2}+\left(\partial^{3} u / \partial x \partial y^{2}\right)^{2}\right] .
$$

Applying the same reasoning to variables $v$ and $w$ leads to the following additional terms for $J_{2}(v)$ and $J_{2}(w)$ :

$$
J_{2}^{\prime}(v)=\mu_{1}\left[\left(\partial^{3} v / \partial y^{3}\right)^{2}+2\left(\partial^{3} v / \partial x \partial y^{2}\right)^{2}+\left(\partial^{3} v / \partial x^{2} \partial y\right)^{2}\right]
$$

$$
J_{2}^{\prime}(w)=\mu_{1}\left[\left(\partial^{3} w / \partial x^{2} \partial z\right)^{2}+\left(\partial^{3} w / \partial y^{2} \partial z\right)^{2}\right.
$$$$
\left.+2\left(\partial^{3} w / \partial x \partial y \partial z\right)^{2}\right] .
$$ 


\section{APPENDIX C}

\section{Discrete Form of the Filter Differential Operator $J_{2}+J_{2}^{\prime}$}

The differential operator $J_{2}+J_{2}^{\prime}$ applied to $u, v$, and $w$ (appendix B) combines a variety of second- to thirdorder derivatives, which are listed below [see (B1), (B8), (B9), and (B10)]:

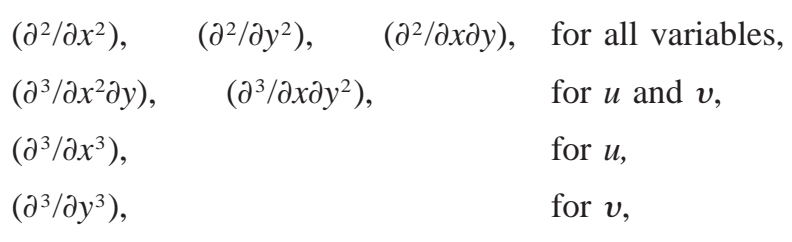

and

$\left(\partial^{3} / \partial x^{2} \partial z\right), \quad\left(\partial^{3} / \partial y^{2} \partial z\right)$

$\left(\partial^{3} / \partial x \partial y \partial z\right), \quad$ for $w$.

Using off-centered finite differences and gridpoint index $I$ in the horizontal and $k$ in the vertical as defined in appendix A, the discrete form of the above derivatives applied to any component $U$ (denoting $u, v$, or $w$ ) can be expressed in the following forms:

$$
\begin{aligned}
\partial^{2} U / \partial x^{2} & =\left(U_{I-1}-2 U_{I}+U_{I+1}\right) / L^{2} \quad \text { at } I . \\
\partial^{2} U / \partial y^{2} & =\left(U_{I-m}-2 U_{I}+U_{I+m}\right) / L^{2} \quad \text { at } I . \\
\partial^{2} U / \partial x \partial y & =\left(U_{I}-U_{I+1}-U_{I+m}+U_{I+m+1}\right) / L^{2}
\end{aligned}
$$

at the center of horizontal grid mesh $I$.

$$
\begin{aligned}
\partial^{3} U / \partial x^{2} \partial y= & \left(-U_{I-1}+2 U_{I}-U_{I+1}+U_{I+m-1}\right. \\
& \left.-2 U_{I+m}+U_{I+m+1}\right) / L^{3}
\end{aligned}
$$

at grid point $I$ shifted by $L / 2$ in the $y$ direction.

$$
\begin{aligned}
\partial^{3} U / \partial x \partial y^{2}= & \left(-U_{I-m}+U_{I-m+1}+2 U_{I}\right. \\
& \left.-2 U_{I+1}-U_{I+m}+U_{I+m+1}\right) / L^{3}
\end{aligned}
$$

at grid point $I$ shifted by $L / 2$ in the $x$ direction.

$$
\partial^{3} U / \partial x^{3}=\left(-U_{I-1}+3 U_{I}-3 U_{I+1}+U_{I+2}\right) / L^{3}
$$

at grid point $I$ shifted by $L / 2$ in the $x$ direction.

$$
\partial^{3} U / \partial y^{3}=\left(-U_{I-m}+3 U_{I}-3 U_{I+m}+U_{I+2 m}\right) / L^{3}
$$

at grid point $I$ shifted by $L / 2$ in the $y$ direction.

$$
\begin{aligned}
\partial^{3} U / \partial x^{2} \partial z=[ & \left(U_{I-1}-2 U_{I}+U_{I+1}\right)_{k} \\
& \left.-\left(U_{I-1}-2 U_{I}+U_{I+1}\right)_{k-1}\right] / L^{2} l
\end{aligned}
$$

at grid point $I$ shifted downward by $l / 2$.

$$
\begin{aligned}
\partial^{3} U / \partial y^{2} \partial z= & {\left[\left(U_{I-m}-2 U_{I}+U_{I+m}\right)_{k}\right.} \\
& \left.-\left(U_{I-m}-2 U_{I}+U_{I+m}\right)_{k-1}\right] / L^{2} l
\end{aligned}
$$

at grid point $I$ shifted downward by $l / 2$.

$$
\begin{aligned}
\partial^{3} U / \partial x \partial y \partial z= & {\left[\left(U_{I}-U_{I+1}-U_{I+m}+U_{I+m+1}\right)_{k}\right.} \\
& \left.-\left(U_{I}-U_{I+1}-U_{I+m}+U_{I+m+1}\right)_{k-1}\right] / L^{2} l
\end{aligned}
$$

at the center of a grid box $I$.

The last three derivatives involve two successive levels $k-1$ and $k$, and components at one specified level are input components, as explained in appendix A. Their expansion into two similar blocks allows one to consider either level $k-1$ or $k$.

\section{APPENDIX D}

\section{Derivation of the Band Diagonal Matrix Equation for MUSCAT}

The discrete form of data fit (8), continuity equation adjustment (A2), and differential operators associated with the filtering process, $(\mathrm{C} 1)-(\mathrm{C} 10)$, should be combined to obtain the final functional $F^{\prime}$ to be minimized with respect to all gridpoint wind components. According to the least squares minimization problem, these components are the solution of a linear system of $3 \mathrm{mn}$ equations that can appear somewhat complex. Indeed, each system equation links the three wind components at several grid points of the horizontal mesh, and a matrix equation is quite useful to represent such a system. In appendixes $\mathrm{A}$ and $\mathrm{C}$, three separate vectors are used to represent the $m n$ grid point values of $u, v$, and $w$. The matrix equation (8) for an individual grid point can be readily expanded to all grid points by using the vector notation: An $m n \times m n$ diagonal submatrix substitutes for each matrix element of (8), while an $m n$ vector replaces each vector element.

Considering a unique vector $\mathbf{U}$ of $3 m n$ elements in place of the successive $m n$-separated $u, v, w$ vector elements, data fit can be expressed in the matrix form

$$
\mathbf{M U}=\mathbf{P}
$$

where $\mathbf{M}$ is a $(3 m n)^{2}$ sparse pentadiagonal (the diagonal plus four subdiagonals) matrix and $\mathbf{P}$ is a $3 m n$ vector associated with observations. Subdiagonals have positions shifted by $\pm m n$ and $\pm 2 m n$ from the main diagonal. Here, $\mathbf{M}$ is a positive definite and symmetric matrix since it is derived from a quadratic positive definite form. Additional terms, described below and resulting from continuity adjustment and filtering, do not modify this property.

The equivalent form of (A2) for an elementary grid box $I$ is

$$
\begin{gathered}
\mathrm{B}_{I}^{\prime}=\left\{a \left(-U_{I}+U_{I+1}-U_{I+m}+U_{I+m+1}-U_{I+m n}\right.\right. \\
\left.\quad-U_{I+m n+1}+U_{I+m n+m}+U_{I+m n+m+1}\right) \\
+b\left(U_{I+2 m n}+U_{I+2 m n+1}+U_{I+2 m n+m}\right. \\
\left.\left.\quad+U_{I+2 m n+m+1}\right)-X_{I}\right\}^{2}
\end{gathered}
$$

It appears that minimizing $\mathrm{B}_{I}^{\prime}$ with respect to any $U$ involves a limited number of grid points and then results 


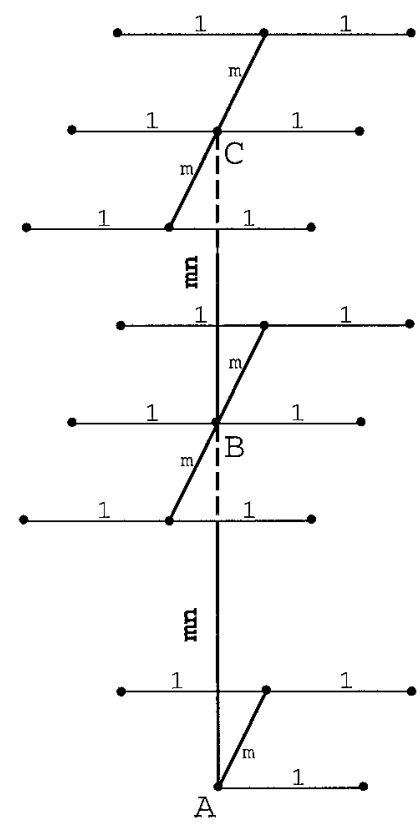

FIG. D1. Schematic 3D representation of the positions (full circles) of the 22 nonzero upper-side (subdiagonals) matrix terms with respect to the main diagonal located on $A$, as deduced from the discrete form of the continuity equation adjustment of MUSCAT. It is based on the gridpoint representation of the $\mathbf{U}$ vector (containing the three successive $m n$ elements of $u, v$, and $w$; see text) in three successive $m n$-separated levels, as illustrated by points A, B, and C. Distance between two grid points connected by a line is reported as the grid number separation (i.e., $1, m$, or $m n$ ). The banded structure is associated with the points connected by horizontal lines composed of successive grid points with a grid number separation of 1 from each other.

in a limited number of nonzero terms, which are the accumulation of $\pm a^{2}, \pm a b$, and $b^{2}$ terms in matrix $\mathbf{M}$, and of $\pm a X$ and $b X$ in vector $\mathbf{P}$.

Positions of nonzero terms that define positions of matrix diagonal and subdiagonals can be readily found by examining the relative positions of the various grid points involved in (D2) with respect to the point for which the minimum of $\mathrm{B}_{I}^{\prime}$ is searched. Relationships of a grid point with itself in (D2) contribute to the diagonal of $\mathbf{M}$. Due to the symmetric property, only positive grid position differences need to be defined. This is facilitated by the increasing arrangement of grid indices in (D2), which allows to examine successively each subscript attached to $U$ with its next. Finally, there are 66 nonzero off-diagonal terms [i.e., $r(r-1) / 2$, where $r$ is the number of grid values included in $\left.\mathrm{B}_{I}^{\prime}\right]$ on the upper side of the diagonal that can be found to be distributed over 22 subdiagonals, the positions of which are shown in Fig. D1. The nearest and the farthest are shifted by 1 and $2 m n+m+1$ ranks from the diagonal. Following Fig. D1 and extending to the symmetric part, minimization of $\mathrm{B}^{\prime}$ over the domain contributes to 45 diagonals that are organized into 15 groups of 3 successive diagonals centered on the diagonal and on $\pm m, \pm(m n-$ $m), \pm m n, \pm(m n+m), \pm(2 m n-m), \pm 2 m n$ and $\pm(2 m n$ $+m)$, respectively.

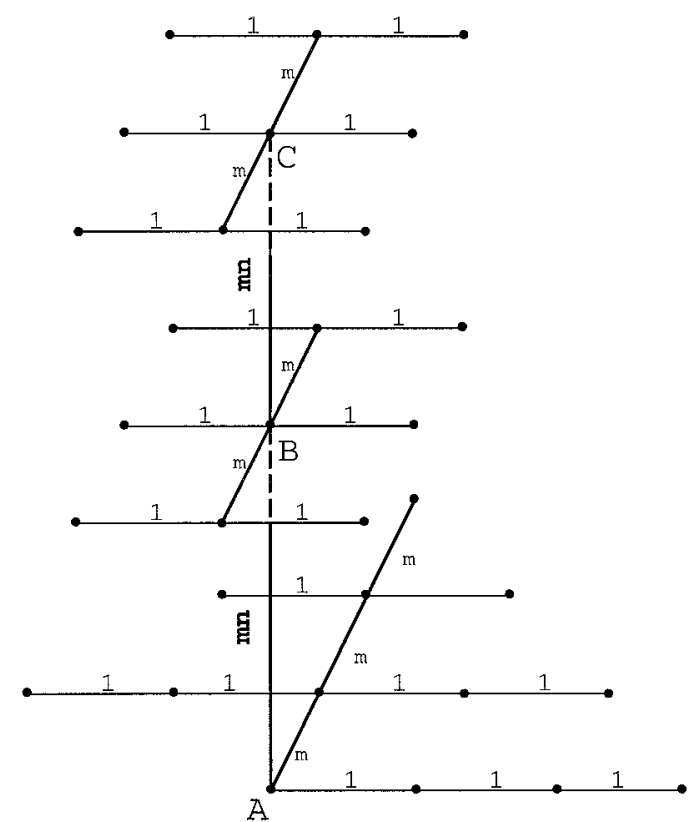

FIG. D2. As in Fig. D1 but for the final structure of the band diagonal matrix associated with the variational form of MUSCAT.

Similarly, it can be found that the differential operators $(\mathrm{C} 1)-(\mathrm{C} 10)$ contribute to the diagonal and 24 subdiagonals at $\pm 1, \pm 2, \pm 3, \pm(m-2), \pm(m-1), \pm m$, $\pm(m+1), \pm(m+2), \pm(2 m-1), \pm 2 m, \pm(2 m+1)$, and $\pm 3 m$.

Finally, Fig. D2 illustrates the final structure of the band diagonal matrix $\mathbf{M}$, consisting of a total of $61 \mathrm{di}-$ agonals organized into 19 groups: a main group of 7 diagonals about the main diagonal, 2 groups of 5 centered at $\pm m, 2$ groups of 3 at $\pm 2 m, 2$ single diagonals at $\pm 3 m$, and 12 groups of 3 at $\pm(m n-m), \pm m n, \pm(m n$ $+m), \pm(2 m n-m), \pm 2 m n$, and $\pm(2 m n+m)$.

\section{REFERENCES}

Chong, M., and J. Testud, 1983: Three-dimensional wind field analysis from dual-Doppler radar data. Part III: The boundary condition: An optimum determination based on a variational concept. J. Climate Appl. Meteor., 22, 1227-1241.

— , and C. Campos, 1996: Extended overdetermined dual-Doppler formalism in synthesizing airborne Doppler radar data. J. Atmos. Oceanic Technol., 13, 581-597.

— squall line from airborne dual-beam Doppler radar data: A test of coplane methodology software. J. Atmos. Oceanic Technol., 13, 36-53.

—, P. Amayenc, G. Scialom, and J. Testud, 1987: A tropical squall line observed during the COPT 81 experiment in West Africa. Part I: Kinematic structure inferred from dual-Doppler radar data. Mon. Wea. Rev., 115, 670-694.

Frush, C. L., P. H. Hildebrand, and C. Walther, 1986: The NCAR airborne Doppler radar. Part II: System design considerations. Preprints, 23d Radar Meteorology Conf., Snowmass, CO, Amer. Meteor. Soc., 151-154.

Gamache, J. F., 1995: A three-dimensional variational method for determining wind velocities from Doppler data as applied to the TOGA COARE test case. Summary Report, TOGA COARE Int. 
Data Workshop TOGA, COARE International Project Office, UCAR, Boulder, CO, 106 pp.

Hildebrand, P. H., and C. K. Mueller, 1985: Evaluation of meteorological airborne Doppler radar. Part I: Dual-Doppler analyses of air motions. J. Atmos. Oceanic Technol., 2, 362-380.

Houze, R. A., Jr., and A. K. Betts, 1981: Convection in GATE. Rev. Geophys. Space Phys., 19, 541-576.

Jorgensen, D. P., T. Matejka, and J. D. DuGranrut, 1996: Multi-beam techniques for deriving wind fields from airborne Doppler radars. J. Meteor. Atmos. Phys., 59, 83-104.

Kabèche, A., and J. Testud, 1995: Stereoradar meteorology: A new unified approach to process data from airborne or ground-based meteorological radars. J. Atmos. Oceanic Technol., 12, 783-799.

Ray, P. S., and K. L. Sangren, 1983: Multiple-Doppler radar network design. J. Climate Appl. Meteor., 22, 1444-1454.

Roux, F., and J. Sun, 1990: Single-Doppler observation of a West African squall line on 27-28 May 1981 during COPT 81: Kin- ematics, thermodynamics, and water budget. Mon. Wea. Rev., 118, 1826-1853.

Sasaki, Y., 1970: Some basic formalisms in numerical variational analysis. Mon. Wea. Rev., 98, 875-883.

Scialom, G., and Y. Lemaître, 1990: A new analysis for the retrieval of three-dimensional mesoscale wind fields from multiple Doppler radar. J. Atmos. Oceanic Technol., 7, 640-665.

Testud, J., and P. Amayenc, 1989: Stereoradar meteorology-A promising technique for observation of precipitation from a mobile platform. J. Atmos. Oceanic Technol., 6, 89-108.

Trier, S. B., W. C. Skamarock, M. A. LeMone, D. B. Parsons, and D. P. Jorgensen, 1996: Structure and evolution of the 22 February 1993 TOGA COARE squall line: Numerical simulations. J. Atmos. Sci., 53, 2861-2886.

Webster, P. J., and R. Lukas, 1992: TOGA COARE: The Coupled Ocean-Atmosphere Response Experiment. Bull. Amer. Meteor. Soc., 73, 1377-1416. 